\title{
GP-SIMD Processing-in-Memory
}

\author{
AMIR MORAD, LEONID YAVITS, and RAN GINOSAR, Technion
}

\begin{abstract}
GP-SIMD, a novel hybrid general-purpose SIMD computer architecture, resolves the issue of data synchronization by in-memory computing through combining data storage and massively parallel processing. GP-SIMD employs a two-dimensional access memory with modified SRAM storage cells and a bit-serial processing unit per each memory row. An analytic performance model of the GP-SIMD architecture is presented, comparing it to associative processor and to conventional SIMD architectures. Cycle-accurate simulation of four workloads supports the analytical comparison. Assuming a moderate die area, GP-SIMD architecture outperforms both the associative processor and conventional SIMD coprocessor architectures by almost an order of magnitude while consuming less power.
\end{abstract}

Categories and Subject Descriptors: C.1.2 [Multiple Data Stream Architectures (Multiprocessors)]; C.1.3 [Other Architecture Styles]

General Terms: Design, Algorithms, Performance, Energy

Additional Key Words and Phrases: Multicore, SIMD, associative processor, processing in memory, PIM

ACM Reference Format:

Amir Morad, Leonid Yavits, and Ran Ginosar. 2015. GP-SIMD processing-in-memory. ACM Trans. Architec. Code Optim. 11, 4, Article 53 (January 2015), 26 pages.

DOI: http://dx.doi.org/10.1145/2686875

\section{INTRODUCTION}

Machine-learning algorithms executed on high-performance computers (HPCs) [Steinkraus et al. 2005] address complex challenges such as mapping the human genome, investigating medical therapies, and executing high-frequency derivative trading. These problems are sequential in the sense that each parallelizable step depends on the outcome of the preceding step, and typically, a large amount of data is exchanged (synchronized) within the chip between sequential and parallel processing cores in each step [Yavits et al. 2014a] (Figure 1). The preferred solution no longer features a single processing unit augmented by memory and disk drives, but a different class of processors capable of exploiting data-level parallelism. SIMD architectures are a class of parallel computers with multiple processing units performing the same operation on multiple data points simultaneously [Intel 2013; AltiVec Engine 2014; ARM 2014; Gschwind et al. 2006; IBM 2005; Sayre 1976]. Such machines exploit data-level parallelism and are thus well suited for machine learning over Big Data [Steinkraus et al. 2005]. High utilization of SIMD processors requires a very high computation-tobandwidth ratio and large datasets [Luebke 2004]. An excess of coarse SIMD computing

This research was funded in part by the Intel Collaborative Research Institute for Computational Intelligence (ICRI-CI) and by the Hasso-Plattner Institute (HPI).

Authors' addresses: A. Morad, L. Yavits, and R. Ginosar, Department of Electrical Engineering, Technion-Israel Institute of Technology, Haifa 3200003, Israel; emails: \{amirm, yavits\}@tx.technion.ac.il, ran@ee.technion.ac.il.

Permission to make digital or hard copies of part or all of this work for personal or classroom use is granted without fee provided that copies are not made or distributed for profit or commercial advantage and that copies show this notice on the first page or initial screen of a display along with the full citation. Copyrights for components of this work owned by others than ACM must be honored. Abstracting with credit is permitted. To copy otherwise, to republish, to post on servers, to redistribute to lists, or to use any component of this work in other works requires prior specific permission and/or a fee. Permissions may be requested from Publications Dept., ACM, Inc., 2 Penn Plaza, Suite 701, New York, NY 10121-0701 USA, fax +1 (212) 869-0481, or permissions@acm.org.

(c) 2015 ACM 1544-3566/2015/01-ART53 $\$ 15.00$

DOI: http://dx.doi.org/10.1145/2686875 


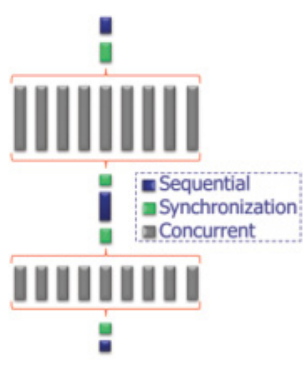

Fig. 1. Typical sequential/parallel processing flow.

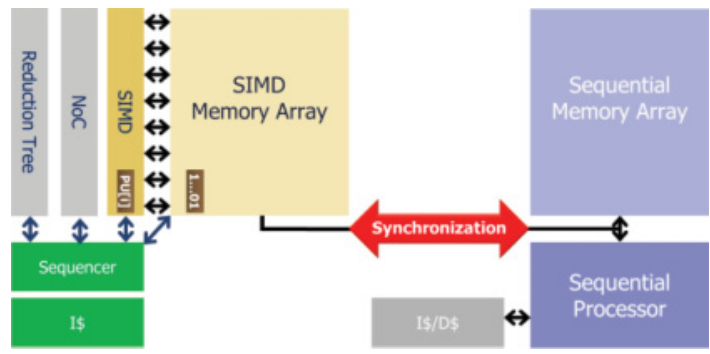

Fig. 2. Synchronization between sequential processor and conventional SIMD coprocessor (CSIMD).

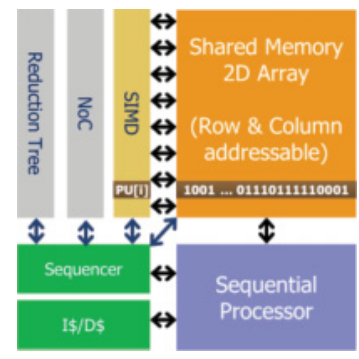

Fig. 3. GP-SIMD conceptual architecture.

elements operating at high rates results in irregular thermal density and hotspots [Sheaffer et al. 2005], further limiting SIMD scalability. Power dissipation and onchip communication are the primary factors limiting the scalability of on-chip parallel architectures [Borkar 2007].

It has been shown [Hill et al. 2008; Morad et al. 2006] that the maximal acceleration of a fine-grained workload containing sequential and concurrent parts occurs when the sequential section is assigned to a large, high ILP sequential processor (containing, for example, accelerators such as a double-precision floating-point unit, function generator, and branch prediction unit), depicted on the right side of Figure 2. The concurrent part is assigned to a massive number of fine-grained low-power processing arrays, shown on the left side of Figure 2. That array is organized as a conventional SIMD (CSIMD). An immediate limiting factor of such architectures is synchronization requiring data exchange [Yavits et al. 2014a] between the sequential processor and the processing array, depicted as a red bus in Figure 2.

In this article, we propose a novel, hybrid general-purpose SIMD computer architecture that resolves the issue of synchronization by in-memory computing through combining data storage and massively parallel processing. Figure 3 details the conceptual architecture of the GP-SIMD processor, comprising a sequential CPU, a shared memory with two-dimensional access, instruction and data caches, an SIMD coprocessor, and an SIMD sequencer. The SIMD coprocessor contains a large number of fine-grained processing units, each comprising a single-bit ALU (for performing bit-serial computations), single-bit function generator, and 4-bit register file. The GPSIMD processor is thus a large memory with massively parallel processing capability. No data synchronization between the sequential and parallel segments is required since both the general-purpose sequential processor and SIMD coprocessor access the very same memory array. Thus, no time and power penalties are incurred for data synchronization between the sequential and SIMD processor. 
The GP-SIMD delivers a number of advantages over CSIMD architecture:

-Data processing and data storage are unified. There is no need for data transfer between sequential memory and SIMD memory.

-GP-SIMD allows concurrent operation of the sequential processor and SIMD coprocessors on the shared memory, allowing the sequential processor to offload a task to the SIMD while continuing to process some other sequential functions.

- The number of GP-SIMD fine-grained processing units matches the number of memory rows, striving to match the entire dataset and obtain maximum parallelism.

- The GP-SIMD architecture enables the sequential processor to associatively address the memory array. It may thus allow reduction of software complexity for certain sequential algorithms.

- GP-SIMD power dissipation is distributed uniformly over the entire processing array rather than being concentrated around a smaller number of large, power-hungry processing cores. Thus, there are fewer hotspots, leading to a further reduction of temperature-dependent leakage power [Banerjee et al. 2003].

The first contribution of this article is a taxonomy categorizing previous works in the processing-in-memory (PIM) and SIMD fields. The second contribution is the novel integration of a fine-grained massively parallel SIMD coprocessor with a standard generalpurpose sequential processor and a novel shared memory with two-dimensional access, leading to improvement in performance as well as reduction in power dissipation. The third contribution of this article is the comparative performance and power analysis of GP-SIMD, a conventional SIMD processor (CSIMD), and an associative processor (AP) [Yavits et al. 2014a], supported by analytical modeling. GP-SIMD performance and power are validated with cycle-accurate simulations. Our research indicates an inflection point where GP-SIMD outperforms CSIMD and AP in both performance and power.

The rest of this article is organized as follows. Section 2 discusses related work. Section 3 provides a detailed description of the GP-SIMD architecture and its operation. Section 4 presents analytical modeling of GP-SIMD performance and power consumption and compares it to a CSIMD processor and to an AP. It also describes cycle-accurate simulations of GP-SIMD. Section 5 concludes this article.

\section{RELATED WORK}

Analytical models of computing architectures (e.g., processing units and interconnection networks) enable design space exploration in a reasonable timeframe [Cassidy and Andreou 2012; Hardavellas et al. 2011; Hill et al. 2008; Loh 2008; Wentzlaff et al. 2010]. Pollack [1999] modeled the performance of modern CPUs as a square root function of the resource assigned to them. Morad et al. [2006] and Hill et al. [2008] augmented Amdahl's law with a corollary to multicore architecture by constructing a model for multicore performance and speedup.

The interactions between multiple parallel processors incur performance overheads. These overheads are a result of synchronization, communication, and coherence costs. Analytical models for these overheads have been studied as well. Morad et al. [2006] modeled the synchronization, communication, and coherence as a time penalty on Amdahl's law, concluding that asymmetric multiprocessors can reduce power consumption by more than two-thirds with similar performance compared to symmetric multiprocessors. Yavits et al. [2014b] studied the overheads and concluded that in applications with high intercore communication requirements, the workload should be executed on a small number of cores, and applications of high sequential-to-parallel synchronization requirements may be better executed by the sequential core. Loh [2008] introduced an extension to Hill and Marty's multicore cost/performance model 


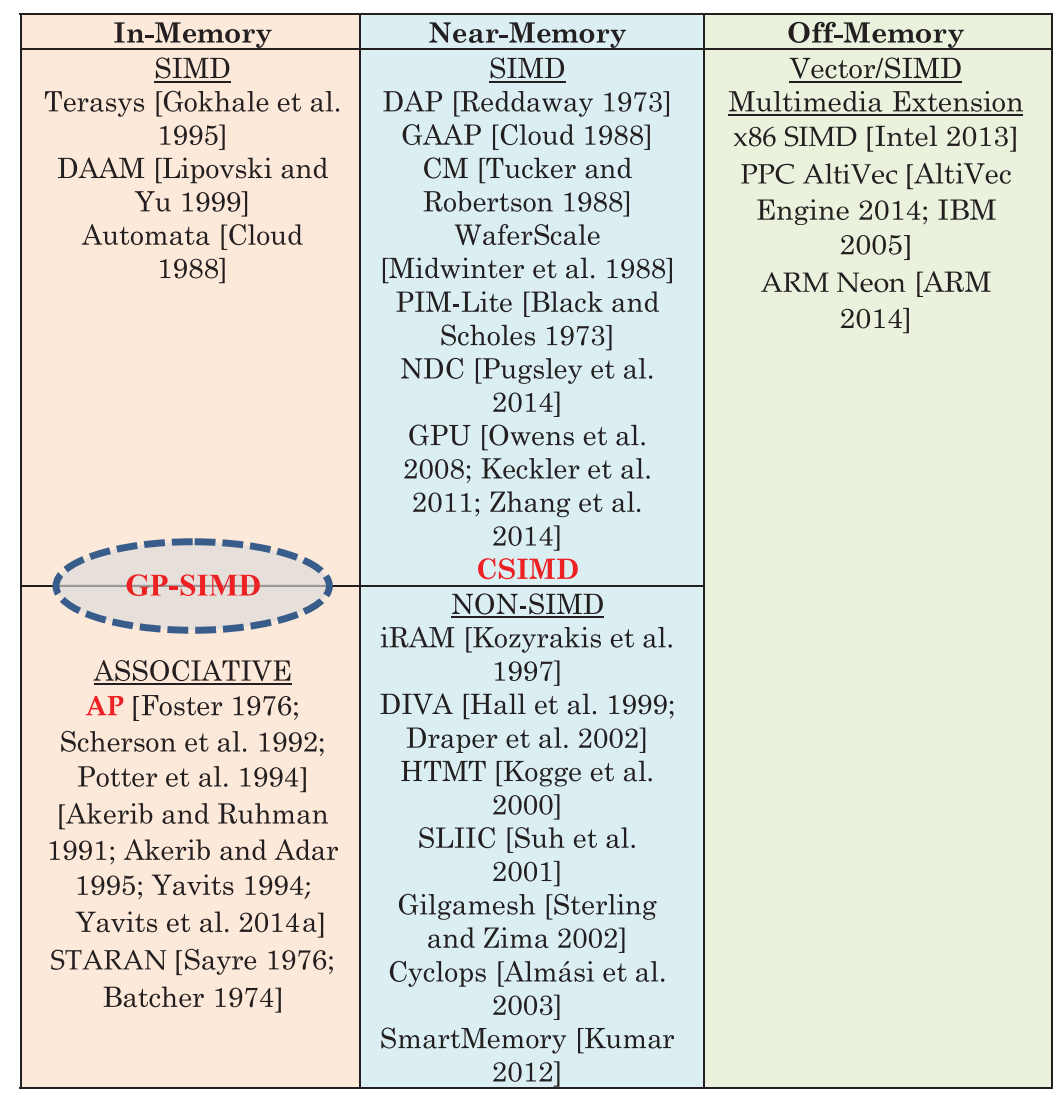

Fig. 4. PiM and SIMD taxonomy.

to account for the "uncore" components, concluding that to sustain the scalability of future many-core systems, the uncore components must be designed to scale sublinearly with respect to the overall core count. Morad et al. [2013, 2014, 2014] presented several frameworks that, given (1) a multicore architecture consisting of last-level cache (LLC), processing cores, and an NoC interconnecting the cores and the LLC; (b) workloads consisting of sequential and concurrent tasks; and (c) physical resource constraints (area, power, execution time, off-chip bandwidth), find the optimal selection of a subset of the available processing cores and the optimal resource allocation among all blocks.

Modeling of SIMD processing performance has been thoroughly discussed. Hong et al. [2009] propose a simple analytical model that estimates the execution time of massively parallel programs. Zhang et al. [2011] develop a microbenchmark-based performance model for NVIDIA GeForce 200-series GPUs.

The concept of mixing memory and logic has been around since the 1970s [Foster 1976]. Similar to Distributed Array Processor (DAP) [Reddaway 1973], STARAN [Batcher 1974; Sayre 1976], CM-2 [Tucker and Robertson 1988], and GAPP [Cloud 1988] computer architectures, GP-SIMD belongs to a PIM class of architectures that use a large number of processing units (PUs) positioned in proximity to memory arrays to implement a massively parallel SIMD computer. To differentiate between GP-SIMD and other works, and since keywords like PIM and SIMD are often used with different meanings in mind, we first classify previous works as follows, and the taxonomy of these works is presented in Figure 4. 
-In-Memory, SIMD: A very large number of small (typically single-bit) SIMD processing units are placed next to the memory, matching the number of memory rows or columns. A single processing unit is allocated per each memory row.

-In-Memory, Associative: A 2D array of associative cells (having compare and write capabilities) are implemented, together with peripheral TAG cells matching the number of memory rows or columns. The bits of an entire memory row (column), together with the TAG, form a single processing unit.

-Near-Memory, SIMD: Many SIMD processing units (usually clustered) are integrated with large blocks of memory. Typically, the processing units incorporate floating-point engines. The number of processing units is much smaller than the number of memory rows.

-Near-Memory, Non-SIMD: Large sequential processors are integrated with large memory blocks. Each processor may also incorporate an off-memory SIMD accelerator.

—Off-Memory, SIMD Accelerators: Several processing units, typically with integer and floating point engines, are operated by main processors.

The In-Memory, SIMD category has been a popular subject of research: Gokhale et al. [1995] designed and fabricated Terasys, a PIM chip, a standard 4-bit memory augmented with a single-bit ALU controlling each column of memory. Lipovski and Yu [1999] introduced a Dynamic Associative Access Memory (DAAM) architecture where a large number of single-bit processing units are put in a DRAM's sense amps. Such DRAM with single-bit ALU delivering arithmetic as well as associative processing is shown to offer nearly three orders of magnitude better cost performance than a conventional microprocessor. Cloud [1988] and Dlugosch et al. [2014] introduced the Automata Processor, a massively parallel processor integrated on a DRAM chip, specifically designed to implement complex regular expression automata for pattern matching.

The In-Memory, Associative category has been thoroughly researched: Foster [1976] detailed an associative processor that combines data storage and data processing and functions as a massively parallel SIMD processor and a memory at the same time. Sayre et al. [1976] and Batcher [1974] discussed STARAN, Goodyear's single-bit SIMD array processor, where the PEs communicate with a multidimensional access (MDA) memory. In bit-slice access mode, associative operations are performed on a single bitslice of all words in parallel, while the word access mode is used in the I/O operations to access a single word so as to allow faster word access to the array from an external data source. Scherson et al. [1992] distributed logic among slices of storage cells such that a number of bit planes share a simple logic unit enabling bit-parallel arithmetic. Potter et al. [1994] presented a parallel programming paradigm called ASC (Associative Computing), offering an efficient associative-based programming model combining numerical computation (such as convolution, matrix multiplication, and graphics) with nonnumerical computing (such as compilation, graph algorithms, rule-based systems, and language interpreters). Akerib and Ruhman [1991] and Akerib and Adar [1995] demonstrated an efficient implementation of several computer vision algorithms on associative processors. Yavits [1994] designed and implemented a stand-alone associative processor chip and studied its performance. Further, Yavits et al. [2014a] presented a computer architecture where an associative processor replaces the last-level cache and the SIMD accelerator, showing performance and power benefits.

The Near-Memory, SIMD category incorporates several notable works. Reddaway [1973] presented a DAP, a $64 \times 64$ single-bit SIMD processing unit (PE) with 4,096 bits of storage per PE. Programs for the DAP were written in DAP FORTRAN with a $64 \times 64$ matrix and 64-unit vector primitives. Cloud [1988] presented the Geometric Arithmetic 
Parallel Processor (GAPP), a two-dimensional array of single-bit SIMD processors, where each processor is allocated with and addresses a distinct memory bank. Tucker and Robertson [1988] presented the Connection Machine's hypercube arrangement of thousands of single-bit SIMD processors, each with its own 4kbits of RAM. Each chip contained a communication channel, 16 processors, and 16 RAMs. The CM-1 employed a hypercube routing network, a main RAM, and an input/output processor. The CM2 added floating-point numeric coprocessors and more RAM. Midwinter et al. [1988] presented a wafer-scale processor containing $128 * 128$ processing SIMD units each consisting of an ALU, 128 bits of local RAM, an input/output register, and a control register. Brockman et al. [2004] evaluated the die cost versus performance tradeoffs of the PIMLite system consisting of a multithreaded core with SIMD accelerator integrated with DRAM, which could serve as the memory system of a host processor, and realized a performance speedup of nearly a factor of 4 on N-Body force calculation. Note, however, that the integration of logic into DRAM cells is neither simple nor efficient due to the different manufacturing process utilized for DRAM. Zhang et al. [2014] explored the usage of $3 \mathrm{D}$ die stacking to move memory-intensive computations closer to memory. A throughput-oriented computing (programmable GPU) showed significantly better performance due to 3D stacking providing increased bandwidth. Pugsley et al. [2014] presented a detailed analysis of in-memory MapReduce in the context of near-data computing (NDC), detailing the insights and quantifying the potential for improvement.

The Near-Memory, Non-SIMD category has been a popular subject of research. Kozyrakis et al. [1997] studied IRAM (intelligent RAM). IRAM utilizes the on-chip real estate for DRAM memory instead of SRAM caches, based on the fact that DRAM can accommodate $30 \times$ to $50 \times$ more data than SRAM. Having the entire memory on the chip, coupled to the processor through an on-chip high-bandwidth and low-latency interface, benefits architectures that demand fast memory I/O. This is clearly limited to applications that require no more data than can fit in that DRAM. Hall et al. [1999] developed DIVA, the Data-Intensive Architecture, combining PIM memories with one or more external host processors and a PIM-to-PIM interconnect. DIVA increases memory bandwidth through performing selected computation in memory, reducing the quantity of data transferred across the processor-memory interface, and provides communication mechanisms for moving both data and computation throughout memory, further bypassing the processor-memory bus. Kogge et al. [2000] proposed to overcome latencies between the main memory and the high-performance CPUs with HTMT, a multilevel memory system using PIM architectures that actively manage the flow of data without centralized CPU control. Suh et al. [2001] presented a PIM-based multiprocessor system, the System Level Intelligent Intensive Computing (SLIIC) Quick look (QL) board. This system includes eight DRAM PIM array chips and two FPGA chips implementing an interconnect network. Sterling and Zima [2002] studied Gilgamesh, an architecture that extends existing PIM capabilities by incorporating advanced mechanisms for virtualizing tasks and data and providing adaptive resource management for load balancing and latency tolerance. The Gilgamesh execution model is based on a middleware layer allowing explicit and dynamic control of locality and load balancing. Almási et al. [2003] introduced Cyclops, an architecture that integrates a large number of processing cores, main memory, and communications hardware on a single chip, and studied the performance of several scientific kernels running on different configurations of this architecture. Kumar [2012] introduced Smart Memory, 2D array of packet processing units, each with local memory array. The PEs are interconnected together via a $2 \mathrm{D}$ mesh NoC. The smart memory chips are aimed at speeding packet processing jobs on large datasets.

Off-Memory Vector/SIMD Multimedia Extension architectures include Intel's x86 SIMD (from MMX to later generations [Intel 2013] (1997), IBM/Motorola/Apple AltiVec 
engine [AltiVec Engine 2014; IBM 2005] (1996), and ARM (from VFP to Neon [ARM 2014] (1985)).

Our proposed GP-SIMD architecture is different from the cited works, as it combines a sequential processor, an in-memory SIMD, an associative coprocessor, and a twodimensional access memory such that (1) the data are shared between the sequential processor and the SIMD coprocessor - that is, the data remain in the same place rather than moved back and forth between the two; (2) the sequential processors and the SIMD coprocessor do not incur penalties due to the shared memory (relative to a single-port memory); and (c) the shared memory array is similar to a standard on-chip single-port memory in terms of area, power, and performance. The GP-SIMD is somewhat similar to the associative processor [Foster 1976]. We compare these two architectures, as well as an abstract GPU like the SIMD processor, in terms of area, energy, and performance and conclude that the GP-SIMD prevails in all criteria.

\section{THE GP-SIMD PROCESSOR}

In this section, we present the GP-SIMD; detail its internal architecture and its arithmetic, logic, and associative processing capabilities; and establish analytical performance and power models. Further, we discuss SRAM-like implementation of the GPSIMD memory array.

\subsection{Top-Level Architecture}

The GP-SIMD is a hybrid general-purpose and SIMD computer architecture that resolves the issue of data synchronization by in-memory computing through combining data storage and massively parallel processing. As illustrated in Figure 5, references to on-chip memory row and column are physical. Each row may contain many words $V_{0}-V_{n}$ of software programmable width. The number of rows typically matches the number of dataset elements, $D$. Each memory row is allocated a single processing unit.

- The sequential processor accesses either one word at a time or multiple words. Typically, such a transaction accesses one physical row at a time.

- The SIMD reads/writes a bit slice (having $r$ bits) composed of the same bit number from all words in some partition of the memory. Physically, it may access multiple bits in a physical row and all rows per access-namely, access multiple columns of the physical array (one after another).

Figure 5 details the architecture of a GP-SIMD processor, comprising the sequential CPU, shared memory array, L1 cache, SIMD coprocessor, SIMD sequencer, interconnection network, and a reduction tree. The sequential processor schedules and operates the SIMD processor via the sequencer. In a sense, the sequential processor is the master controlling a slave SIMD coprocessor. Any common sequential processor may be used, be it a simple RISC or a complicated processor. At the very least, the selected processor should execute normal sequential programs. The SIMD coprocessor contains $r$ fine-grained bit-serial processing units (PUs), depicted in Figure 6, each containing a single bit Full Adder ("+"), single bit Function Generator ("Logic"), and a 4-bit register file, $R A, R B, R C$, and $R D$. A single PU is allocated per row of the shared memory array and physically resides close to that row. The PUs are interconnected using an interconnection network (discussed in the context of Figure 9). The set of all row registers of the same name constitute a register slice. Note that the length of the memory row (e.g., 256 bits) may be longer than the word length of the sequential processor (e.g., 32 bits), so that each memory row may contain several words.

Note the key architectural differences between CPUs and GPU/APUs and GP-SIMD: while CPUs and GPU/APUs only share a memory, the GP-SIMD also enables inmemory processing (PIM). Further, while CPUs and GPU/APUs can only access their 


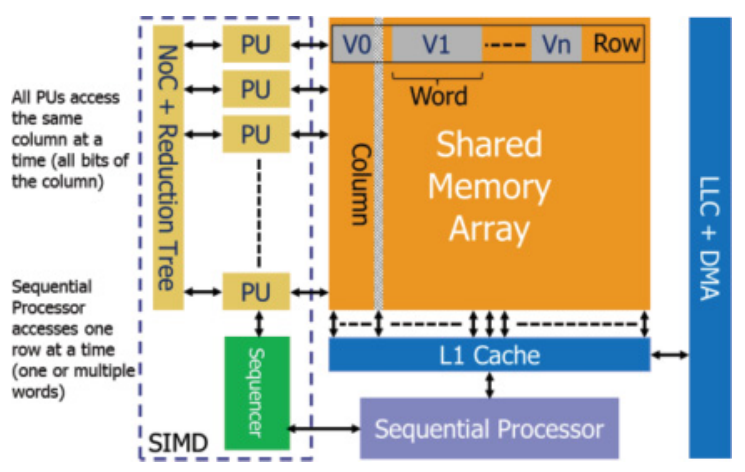

Fig. 5. GP-SIMD top-level architecture.

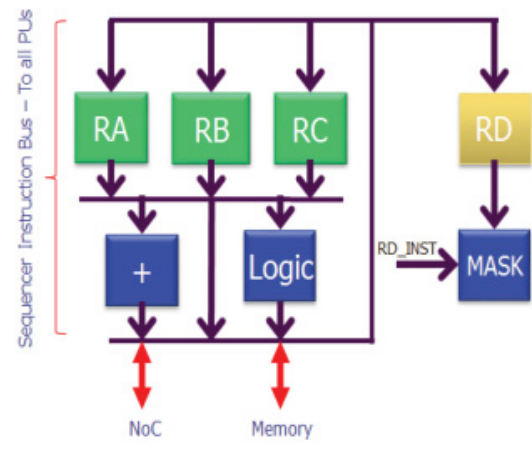

Fig. 6. GP-SIMD processing unit.

Table I. Conditional/Masked PU Microinstructions

\begin{tabular}{lcl}
\hline RD_INST & RD Value & \multicolumn{1}{c}{ Operation } \\
\hline 00 & 0 & $\begin{array}{l}\text { Memory access (read/write) by the sequential processor or the SIMD } \\
\text { coprocessor } \\
\text { Conditional read command, according to an RD: }(R D>0 ? R B=0: R B= \\
\text { Memory output) }\end{array}$ \\
\hline 01 & 1 & $\begin{array}{l}\text { SIMD coprocessor memory-write of RA } \\
\text { Conditional write command, according to } R D:(R D>0 \text { ? Memory input }=R B: \\
\text { Memory input }=R A)\end{array}$ \\
\hline 10 & 0 & $\begin{array}{l}\text { Conditional (masked) read/write: disable row for memory access by sequential } \\
\text { processor. Used during associative access } \\
\text { Conditional (masked) read/write: enable row for memory access by sequential } \\
\text { processor. Used during associative access }\end{array}$ \\
\hline
\end{tabular}

shared memory in a word-sequential manner, the SIMD of GP-SIMD accesses memory in a word-parallel manner, reaching all memory words at once. In addition, while current CPUs and GPU/APUs access their shared memory through a multilevel cache hierarchy, the SIMD in GP-SIMD accesses the shared memory directly and no transfers to cache are needed. As a result of these differences, Morad et al. [2014] details an advantage of power efficiency (GFLOPS/W) relative to such conventional architectures.

When the SIMD reads data from the shared memory, the contents of a bit slice of the memory are transferred into the register slice $(R A s, R B s$, or $R C s)$. Upon writing to the shared memory by the SIMD, the contents of one of the register slices are transferred into the GP-SIMD memory array. The PU is controlled by microinstructions that are broadcast by the sequencer to all PUs. Some operations are conditioned by the singlebit register $R D$, as determined by a two-bit field of the microinstruction RD_INST . The conditional operations are listed in Table I. These conditional microinstructions are handy for implementation of a variety of algorithms (e.g., in Section 4.5). While the first four operations are self-explanatory, the last two operations allow the sequential processor to perform associative commands on the memory array, as detailed in Section 3.4.

\subsection{Operating Modes}

The GP-SIMD may be operated in three modes:

-Sequential Processing Mode, in which the general-purpose sequential processor accesses data in memory during the execution of the sequential segments of a workload; data is typically transferred in lines to and from L1 cache (when the L1 cache line is longer than the row in shared memory, a single line from L1 spans multiple rows). 
—SIMD Processing Mode, in which the parallelizable segments of a workload are executed. The SIMD addresses a column of the memory and reads or writes to all rows of memory. Note that each PU corresponds to a single memory row. Thus, in a single SIMD memory access, all PUs simultaneously read from or write into a single column of the memory array.

- Concurrent Mode, in which both the sequential processor and the SIMD coprocessor can access the shared memory.

In contrast with typical operation of Off-Memory SIMD accelerators, the sequential and SIMD processors may operate concurrently. In such a case, the sequential processor issues an SIMD instruction to the SIMD sequencer in a nonblocking manner and proceeds to execute its own sequential code. The WAIT() synchronization instruction waits for the sequencer to complete a pending sequence of SIMD instructions before the sequential processor can proceed. There are no coherency issues (as there are no race conditions). The hardware does not provide special means; rather, the software is designed to enable noninterfering operation of both the CPU and SIMD, as demonstrated in Morad et al. [2014]. The memory block is divided into a shared memory segment, which is shared by the sequential processor and the SIMD, and an SIMD-only segment, which is accessible only by the SIMD. Data does not need to be transferred by the SIMD to the CPU. Rather, shared data is created in the shared segment, either by the SIMD or the CPU. Thus, no data synchronization between sequential and parallel segments is required since both the sequential processor and SIMD coprocessor access the very same memory array. Consequently, time and power are no longer incurred for data synchronization.

\subsection{Arithmetic/Logic Operations}

GP-SIMD can implement a wide range of arithmetic and logic processing tasks. Consider a workload using two datasets, $A$ and $B$, each containing $D$ elements, where each element is $m$ bits wide. These vectors are mapped into the GP-SIMD memory array such that two $m$-bit-adjacent column-groups hold vectors $A$ and $B$, such that each memory row holds a single element of $A$ and $B$. Assume that we need to add the two vectors and place the results into $m+1$-bit column-group $S$, as illustrated in Figure 7 (where $m=4$ ). The addition is performed in $m$ single-bit addition steps:

$$
c[*] \mid s[*]_{i}=a[*]_{i}+b[*]_{i}+c[*] \quad \forall i=0, \ldots, m-1,
$$

where $i$ is the bit index and $*$ indexes a PU and a memory row. A bitwise vector addition of datasets having four elements $(D=4)$ of 4 bits word length $(m=4)$ is shown in Figure 8. Six cycles are demonstrated in six subfigures. Each subfigure illustrates the PU registers $R A, R B$, and $R C$ and the memory array with the operands $A$ and $B$ and the output $S$. In cycle 1 , column $A[0]$ is copied into $R A$ and $R C$ is reset to all zeroes. In cycle 2 , column $B[0]$ is copied into $R B$. In cycle $3, R A$ and $R B$ are added; the sum is written into $R B$ and the carry replaces $R C$. In addition, column $A[1]$ is read into $R A$. In cycle 4 , the sum in $R B$ is copied to $S[0]$. In cycle 5 , column $B[1]$ is read into $R B$. This process repeats in subsequent cycles. Since addition is carried out simultaneously for all vector elements, fixed-point $m$-bit addition consumes $3 m \in O(m)$ cycles, independent of the size of the vector $D$. Note the contrast with CSIMD architectures having $k$ PUs where $k \ll D$, such as two to 16 PU SIMD accelerators in CPUs [ARM 2014]; they require $\mathrm{O}(D / k) \in O(D)$ cycles to add $D$ data elements.

Fixed-point multiplication and division in GP-SIMD are also implemented bit serially but word parallel, consisting of a series of add-shift and subtract-shift vector operations. Shift is implemented by appropriate column addressing and therefore requires no extra cycles. Thus, fixed-point $m \times m$ bit vector multiplication requires $3 m * m \in O\left(m^{2}\right)$ cycles, 


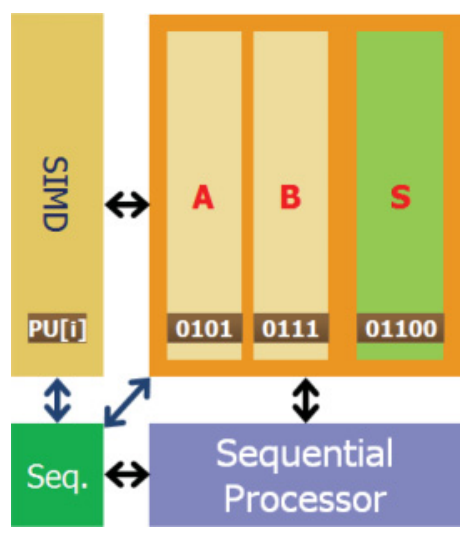

Fig. 7. Memory array containing three operands $(m=4)$.

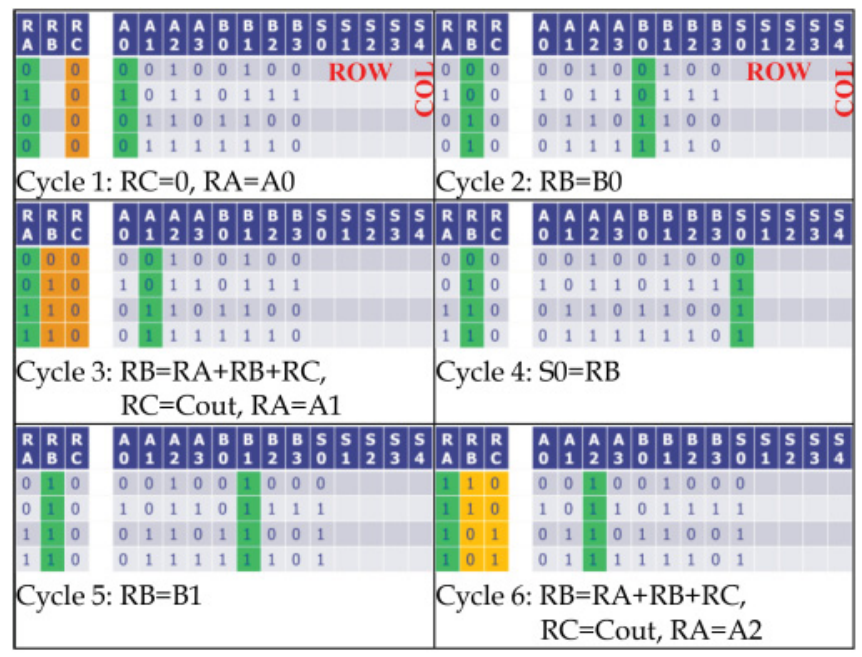

Fig. 8. Addition, cycle by cycle.

regardless of the vector size, $D$. Floating-point arithmetic for GP-SIMD is somewhat more complex to implement. IEEE single-precision floating-point vector multiplication takes close to 2,500 cycles, regardless of the length of the dataset, $D$ (assuming the entire dataset fits into GP-SIMD memory).

In this article, we consider the single-bit processing unit and single-bit SIMD memory access. However, the processing unit and memory access may employ a larger word length. In such a case, the following considerations may apply:

-If the PU is made $x$-bit parallel with $y$-bit-parallel memory access, the twodimensional memory array should increase, as typical memory rows are single bit high, allowing very few horizontal wires. Note further that $y$-bit SIMD access to memory complicates sequential processor memory access as a single word is mapped into $y$ separate rows.

-If the PU is made $x$-bit parallel with serial (1-bit) memory access, its sheer size may not allow placing it in the form factor of memory rows. Further, the PU hardware may become heavily underutilized due to waiting for data.

\subsection{Associative Operations}

GP-SIMD, besides being a massively parallel SIMD accelerator, can implement classical CAM operations such as associative search, sorting, and ordering. The CAM allows comparing all data words to a key, tagging the matching words, and possibly reading some or all tagged words one by one. Consider a large vector, where each element is $m$ bits wide, illustrated by column $A$ in Figure 7. The sequential processor wishes to find all elements in vector $A$ matching a certain Key of $m$ bits and reset the matched values of $A$ (i.e., $A[i \mid A[i]==K e y]=0$ ). The sequential CPU issues a compare of Key on column $A$, storing the single bit-slice compare results output in register $R D$. At this point, register $R D$ has logic one in the rows where $A$ matches the Key and zero elsewhere. Next, a masked write is performed by the sequential processor only to flagged rows of the memory array. To that end, $R D$ enables writing of each memory row (RD_INST = "10," cf. Table I), and the sequential processor writes " 0 " to the $A$ column of the memory array. Only the matching rows are enabled for writing, and the A values of only these rows are reset. Elsewhere, in nonmatching rows, the A values are left unaffected. 


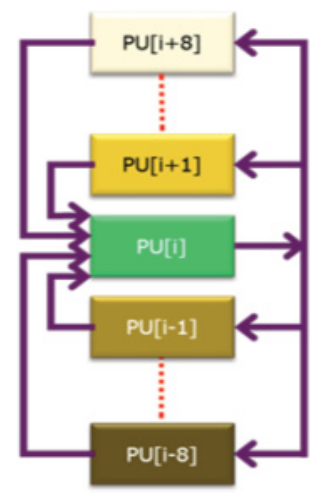

Fig. 9. GP-SIMD $\pm \mathrm{k}$ nearest neighbor interconnection network: each PU is interconnected with nearest neighbors $\pm 1, \pm 2, \ldots, \pm 8$.

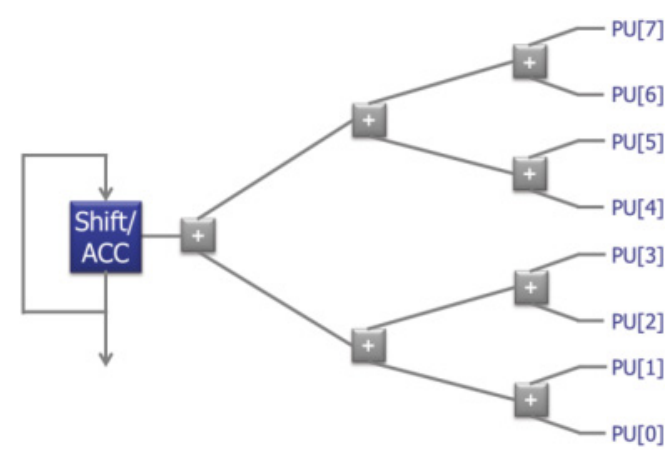

Fig. 10. Hardware reduction tree, illustrated for eight PUs.

Content-addressable access is achieved as follows. Assume that the memory array contains a vector of unique indices $(A)$, adjacent to a vector of data $(B)$. Comparing vector A with a key, followed by setting the RD_INST Bus to "10" while issuing read to the memory array, allows the sequential processor to fetch a single value of vector $B$, corresponding to the row in which vector $A$ matched the Key (i.e., Output $=B[i \mid A[i]$ $==K e y]$ ). When multiple rows match the key, the values must be read one by one. The associative commands have proved valuable for efficient implementation of, for example, sparse matrix multiplication [Morad et al. 2014]. In addition, GP-SIMD can also implement a TCAM.

\subsection{Interconnection Network}

The arithmetic, logic, and associative operations presented earlier require the relevant operands to be available at the memory row of the PU. However, common workloads require inter-PU data communications. Depending on the workload, communication requirements may vary from no communication (for "embarrassingly parallel" tasks such as Black-Scholes option pricing) to relatively intense communications (e.g., for FFT). In some cases, support for special predefined communication patterns or permutations can be useful (e.g., the permutations required for FFT). A dedicated interconnect is employed to allow all PUs to communicate in parallel.

Since the GP-SIMD processing operation is mainly bitwise, the interconnection can be a relatively simple circuit-switched network. An example of an efficient network is a \pm 8 nearest neighbor, as in Figure 9. Each PU has a single-bit direct access to its $\pm Y$ neighbors, where $Y \in\{1,2,4,8\}$. Transferring in parallel an entire vector of $D$ rows by $H$ rows up or down entails approximately $H / 8$ cycles, independent of the vector size, $D$.

\subsection{Reduction Tree}

A common reduction operation sums up a large array of values. Other common reduction operations are minimum or maximum. Reduction tree [Qing et al. 2013], (earlier introduced as a "response counter" in Yavits [1994]) is an adder tree, enabling bitserial parallel summation of PU values. Consider a vector $A$ of $D$ fixed-point $m$-bit elements, as illustrated in Figure 7. Further, consider a hardware reduction tree implemented using a pipelined binary adder tree. The first level of the tree sums two single bits from two adjacent PUs. Following $\log _{2} N$ levels, the scalar sum of the entire array becomes available, as illustrated in Figure 10. The fixed precision summation 


\begin{tabular}{|c|c|c|}
\hline A & T & S \\
\hline 1 & & 1 \\
\hline 2 & & 2 \\
\hline 4 & & 4 \\
\hline 8 & & 8 \\
\hline 16 & & 16 \\
\hline 32 & & 32 \\
\hline 64 & & 64 \\
\hline \multicolumn{3}{|c|}{$\begin{array}{c}\text { Step-1: } \\
\text { S=A }\end{array}$} \\
\hline \multicolumn{3}{|c|}{}
\end{tabular}

\begin{tabular}{|c|c|c|}
\hline $\mathbf{A}$ & $\mathbf{T}$ & $\mathbf{S}$ \\
\hline 1 & 12 & 15 \\
\hline 2 & 24 & 30 \\
\hline 4 & 48 & 60 \\
\hline 8 & 96 & 120 \\
\hline 16 & 64 & 112 \\
\hline 32 & 0 & 96 \\
\hline 64 & 0 & 64 \\
\hline
\end{tabular}

\begin{tabular}{|c|c|c|}
\hline A & $\mathbf{T}$ & $s$ \\
\hline 1 & 112 & 127 \\
\hline 2 & 96 & 126 \\
\hline 4 & 64 & 124 \\
\hline 8 & 0 & 120 \\
\hline 16 & 0 & 112 \\
\hline 32 & 0 & 96 \\
\hline 64 & 0 & 64 \\
\hline
\end{tabular}

Fig. 11. Software reduction tree, illustrated for eight PUs.

of vector $A$ entails reading a single column slice of vector $A$, LSB first, and summing this column via the reduction tree. The addition is carried out simultaneously for all vector elements, one column slice at a time, until all $m$ columns have been processed. The $m$ outputs of the adder tree are summed together using an accumulator, where in each summation loop, the output of the adder tree is shifted left, corresponding to the bit location being processed. Therefore, fixed-point $m$-bit reduction entails $O\left(m+\log _{2}(N)+1\right) \in O\left(m+\log _{2}(N)\right)$ cycles. Partial sums in the intermediate levels are also available to the sequential processor; this is used, for instance, in DMM (Section 4.5).

The reduction tree can also be implemented in software using the PUs and the interconnection network. Consider, for example, a vector $A$ of eight fixed-point 7-bit elements, as in Figure 11. Vectors $T$ and $S$ contain partial sums. Similarly to the hardware implementation, the parallel binary tree is implemented as follows: in the first (initialization) step, vector $A$ is copied onto $S$. In the second step, vector $S$ is shifted one row up and stored in vector $T$, followed by adding vectors $T$ and $S$. The oddly addressed elements of vector $\mathrm{S}$ contain the results of the first step of the binary tree addition. In the third step, vector $S$ is shifted two rows up and stored in vector $T$, followed by adding vectors $T$ and $S$. Vector $S$ now contains the results of the second level of the addition tree. In the fourth step, vector $S$ is shifted four rows up and stored in vector $T$, followed by adding vectors $T$ and $S$. Vector $S$ now contains the results of the third level of the tree, and the sum of all elements of A appears in the first element of $\mathrm{S}$.

In general, given a $\pm \log _{2} N$ interconnection network, fixed-point $m$-bit reduction entails $O\left(2 m+(2 m+3 m) \log _{2}(N)\right) \in O\left(m \log _{2}(N)\right)$ cycles. In case of large datasets and limited interconnection networks, the last few shifting and summation steps can be executed serially by the sequential processor so as to save processing time and power.

\subsection{Circuit Implementation}

The GP-SIMD PU utilizes its memory row as its register file for storing variables and temporaries. The width of the memory array is much higher than the word width of the sequential processor (e.g., 256 distinct columns vs. 32-bit words). A typical GPSIMD memory array is depicted in Figure 12. To enable 2D access (word access by the sequential processor and column access by the SIMD coprocessor), two types of cells are proposed. A shared-memory cell consisting of a seven-transistor SRAM bit is used in the shared columns (Figure 13), and an SIMD-Only cell using a five-transistor cell is used in the SIMD-only columns (Figure 14).

The standard 6T SRAM bit cell (blue in Figure 13) is amended by a pass-gate to enable column read/write access (red). In the SIMD-only static bit cell (Figure 14), a 4T latch (blue) is connected by a pass-gate (red) to enable column read/write access. The bit_line, bit_line_not,word_line, and associated pass-gates of Figure 13 are eliminated 


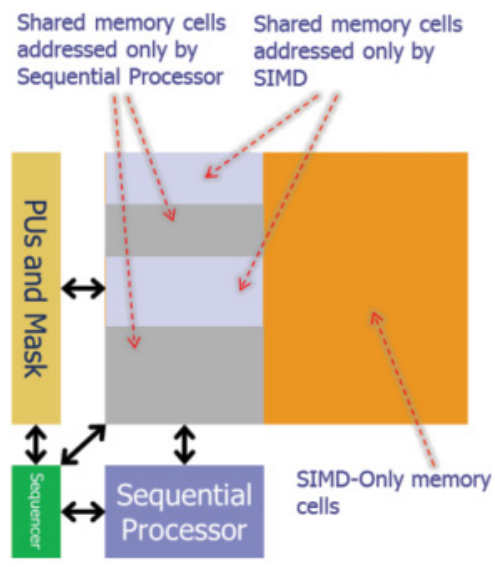

Fig. 12. GP-SIMD memory segmentation.

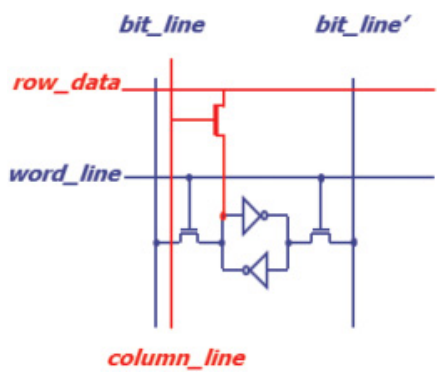

Fig. 13. Shared cell.

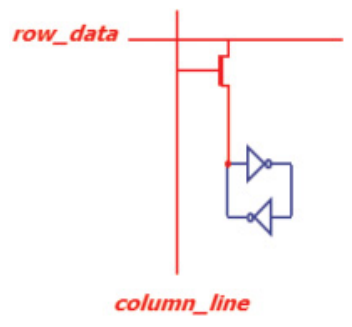

Fig. 14. SIMD-only cell.

in Figure 14 as the sequential processor does not address these cells. Reading and writing to the two-dimensional memory array is performed as follows:

-By sequential processor: to read data from memory, the bit_line and bit_line-not lines are precharged, the word_line is asserted, and the bit lines are sensed. To write data to the memory, the bit_line, bit_line-not, and word_line are asserted.

-By SIMD coprocessor: to read data from memory, the column_line is asserted, and the row_data is precharged. To write data to the memory, the column_line and the row_data are asserted.

When the width of the memory is sufficiently small (e.g., 256 columns), a single pass-gate transistor is tied to the row data. In wider arrays, a differential pair, namely, row_data and row_data-not, and an additional pass-gate transistor may be required. GP-SIMD's 6T and 5T SRAM cell outweigh the AP 12T cell of Yavits et al. [2014a] both area- and power-wise. For the same die area, the GP-SIMD array thus packs nearly twice the number of memory rows and offers twice the AP performance for the same die area. These cells have been validated with Spice and demonstrate no degradation in performance versus the standard SRAM cell. There are several alternatives to placing the PUs alongside the memory rows: (1) customized layout positioning of the PU within the pitch of the memory rows, specifically since the size of the PU is relatively small; (2) $k$ PUs placed side by side next to k memory rows, as in Figure 16; and (3) PUs placed on both sides of the memory block. To account for possible place and route mismatch, the analysis in Section 4 reserves 15\% for spare area.

The memory architecture enables the sequential processor and the SIMD coprocessor to concurrently read from and write to the shared memory. Further, the architecture inherently allows segmentation of the memory array along both dimensions so as to avoid conflict and allow processing on parts of the data, as follows:

- Segmentation in the horizontal dimension: since the memory width is larger than the sequential processor data-bus (e.g., 64-bit data-bus and 256-bit wide array), the SIMD coprocessor may be programmed to read from the entire memory array but write only to the SIMD-only section while the sequential processor continues to process the shared memory space.

- Segmentation along the vertical dimension: $R D$ can be used to mask the word_line of certain rows. 


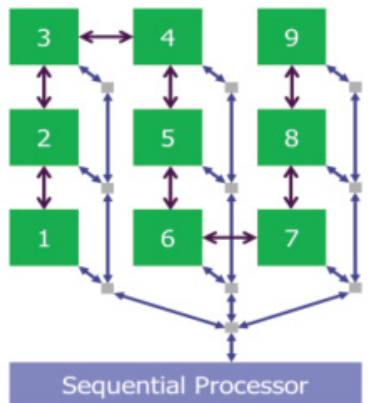

Fig. 15. Array partitioning.

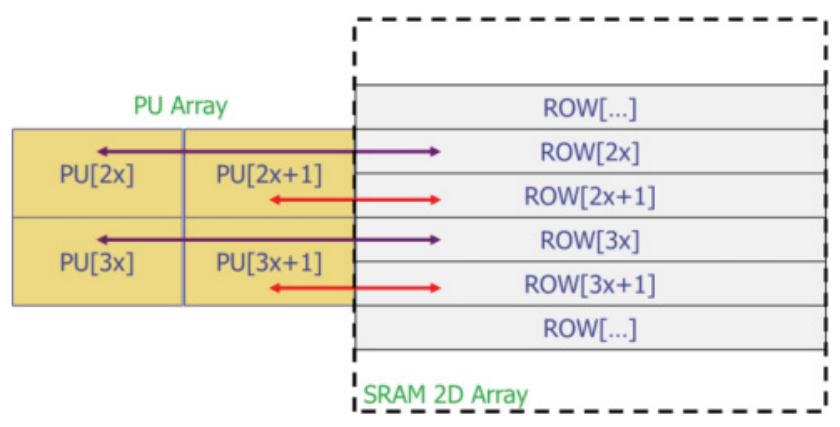

Fig. 16. Staggered PU layout.

-Full segmentation: access by the sequential processor and the SIMD coprocessor may be interleaved to avoid all interference.

The sequential processor may not have access to the entire SIMD memory. Thus, the software must ensure minimal transfers between the shared and SIMD-only segments. Since the number of memory rows in a typical GP-SIMD implementation could be quite high (above 1 million), the GP-SIMD array can be partitioned into $n$ smaller blocks each having $D / n$ processors (and $D / n$ complete memory rows). Each block could have, for example, its own sequencer. In such a case, all blocks share a piped version of the bus to the sequential processor. A potential layout of such an implementation is depicted in Figure 15, in which a GP-SIMD array is partitioned into nine smaller blocks. Note the connectivity simplicity: the interconnection network is chained (red), and the sequential processor memory bus (blue) is connected to all blocks in parallel. Note that since the sequential processor accesses no more than a single memory row at a time, such access involves only a single GP-SIMD block and the path to it; only that path is activated to conserve power. Reading data from a GP-SIMD block could entail a few cycles due to the long path the signal may have to traverse. To mitigate such access delays, the sequential processor includes a (write-through) data cache. If cache hierarchy exists, the shared memory array having a designated and distinct address space will connect to the sequential processor's L1 cache (see Figure 5). While the sequential processor may retain a typical cache hierarchy, data that needs SIMD processing may be fetched directly into the shared memory, bypassing L1. Since most processing is done in the shared memory array, the GP-SIMD architecture may not incorporate a higher level of cache hierarchies beyond L1. As described in Morad et al. [2014], 8 million rows of PUs, each having 256-bit memory rows, and a reasonably sized sequential processor would occupy close to $200 \mathrm{~mm}^{2}$ in $22 \mathrm{~nm}$ technology.

\subsection{GP-SIMD Programming Model and Performance}

The SIMD coprocessor is controlled by its sequencer. The sequencer converts each SIMD instruction into a sequence of microinstructions for the SIMD PUs. The SIMD instruction set is listed in Table II, and the special sequential processor instruction set is listed in Table III. In contrast with typical operation of Off-Memory SIMD accelerators, the sequential and SIMD processors of GP-SIMD may operate concurrently. The sequential processor issues an SIMD instruction to the SIMD sequencer in a nonblocking manner and proceeds to execute its own sequential code. Naturally, the sequencer does not process an instruction prior to completing the previous one. The WAIT() instruction waits for the sequencer to complete its pending sequence of SIMD instructions before the sequential processor can proceed. Table II also summarizes the arithmetic/logic 
Table II. SIMD Instruction Set

\begin{tabular}{|c|c|c|}
\hline Instruction & Type & Performance (cycles) \\
\hline$\overline{\mathrm{INV}}$ (SRC, DST) & Logic & $(1+2 m) \in O(m)$ \\
\hline $\begin{array}{l}\text { AND / OR / XOR (SRC1, SRC2, } \\
D S T)\end{array}$ & Logic & $(1+3 m) \in O(m)$ \\
\hline $\begin{array}{l}\text { ANDI / ORI / XORI (SRC1, SRC2, } \\
D S T)\end{array}$ & Logic (Imm.) & $(1+2 m) \in O(m)$ \\
\hline $\begin{array}{l}\text { ADD / SUB_FIXED (SRC1, SRC2, } \\
m, D S T)\end{array}$ & Arith., Fixed Point & $(1+3 m) \in O(m)$ \\
\hline $\begin{array}{l}\text { ADD / SUB_FP (SRC1, SRC2, } \\
D S T)\end{array}$ & Arith., FP & 600 \\
\hline $\begin{array}{l}\text { MUL / DIV_FIXED (SRC1, SRC2, } \\
m, D S T)\end{array}$ & Arith., Fixed Point & $m(1+3 m) \in O\left(m^{2}\right)$ \\
\hline MUL / DIV_FP (SRC1, SRC2, DST) & Arith., FP & 2500 \\
\hline $\begin{array}{l}\text { UP_/ DOWN_FIXED (SRC, NUM, } \\
m, \bar{D} S T)\end{array}$ & NoC., Fixed Point & $(1+2 m) \in O(m)$ \\
\hline UP / DOWN_FP (SRC, NUM, DST) & NoC., FP & 65 \\
\hline CPY_FIXED $(S R C, m, D S T)$ & Arith., Fixed Point & $(2 m) \in O(m)$ \\
\hline CPY_FP $(S R C, D S T)$ & Arith., FP & 64 \\
\hline RED_SW_FIXED (SRC, $m, D S T)$ & Reduction, SW, Fixed & $(1+3 m+1+2 m) \log _{2} N \in O(m \log N)$ \\
\hline RED_SW_FP $(S R C, D S T)$ & Reduction, SW, FP & $(600+1+64) \log _{2} N \in O(\log N)$ \\
\hline RED_HW_FIXED (SRC, $m, D S T)$ & Reduction, HW, Fixed & $\left(1+m+\log _{2} N\right) \in O(m+\log N)$ \\
\hline RED_HW_FP (SRC, DST) & Reduction, HW, FP & $\left(1+32+\log _{2} N\right) \in O(\log N)$ \\
\hline READ_RD $(C O L)$ & Read a col into $R D$ & 1 \\
\hline READ_MASK (COL) & Masked Read & 1 \\
\hline WRITE_MASK $(C O L, D A T A, m)$ & Masked Write & 1 \\
\hline READ_COND $(C O L, D A T A, m)$ & Seq. Associative Read & 1 \\
\hline WRITE_COND $(C O L, D A T A, m)$ & Seq. Associative Write & 1 \\
\hline COMPARE $(D A T A, C O L, m)$ & Associative Compare & $(1+m) \in O(m)$ \\
\hline
\end{tabular}

$S R C x=$ source column (LSB of the variable's source); $D S T=$ destination column (LSB of the variable's destination); FIXED = fixed-point arithmetic; $F P=$ floating-point arithmetic.

Table III. Sequential Processor Special Instruction Set

\begin{tabular}{l|c|c}
\hline Instruction & Type & Performance (cycles) \\
\hline SP_READ $(R O W, C O L, m)$ & Seq. Proc. Read & 1 \\
SP_WRITE $($ ROW, COL, DATA, $m)$ & Seq. Proc. Write & 1 \\
WAIT( $)$ & Synchronization & 1, minimum \\
\hline
\end{tabular}

performance of the SIMD coprocessor. The SIMD instruction sequences are either programmed manually or generated by compilers. Typically, SIMD operations should be made available as library functions.

Due to the novel bit cell of the GP-SIMD shared memory array (Figures 13 and 14), $S P \_R E A D$ and SP_WRITE may be executed concurrently with SIMD operation. The software should avoid writing conflicts by both processors to the same memory rows and columns. Programming examples are provided in Morad et al. [2014].

\section{ANALYTIC MODEL AND COMPARATIVE ANALYSIS}

In this section, we provide an analytical performance and power consumption model of the CSIMD, AP, and GP-SIMD and compare their relative performance, area, and power consumption under constrained area and power resources. For the purpose of modeling, we assume that the sequential processor is identical in all architectures and focus on concurrent workloads. We further assume that the CSIMD executes a single fused multiply-add operation in $1.5 \mathrm{GHz}$, while the GP-SIMD and AP employ faster clocks of $2.5 \mathrm{GHz}$, as they execute bitwise rather than word-parallel instructions and thus have shorter critical paths. 
Consider a workload having WL single-cycle instructions (i.e., arithmetic, control, register file access, etc.). The workload consists of $N$ fine-grained concurrent execution segments, requiring $T_{R e f}=W L * 1$ cycles to execute on a baseline reference floatingpoint engine capable of performing $1 \mathrm{FLOP} /$ cycle (single-precision floating-point operation per cycle). Such a floating-point engine consumes an area of $0.01 \mathrm{~mm}^{2}$ in $45 \mathrm{~nm}$ [Pedram 2013] and dissipates $10 \mathrm{~mW}$ [Keckler et al. 2011]. With 22nm process technology, the same floating-point engine would consume an area of $0.003 \mathrm{~mm}^{2}$ and dissipate roughly $5 \mathrm{~mW}$ [Cassidy and Andreou 2012; Keckler et al. 2011]. These figures pertain to a baseline floating-point engine, without the accompanying connectivity logic, register file, and the like.

A portion of the workload instructions, $C_{S y n c}$, is dedicated to data synchronization before and after each segment starts and ends, respectively, while a further portion of the workload, $C_{\text {Inter }}$, is dedicated to data exchange among the execution units. The remaining portion of the workload, $C_{P r o c}$, is actual processing. We thus have

$$
C_{\text {Proc }}+C_{\text {Sync }}+C_{\text {Inter }}=1 .
$$

In a large design composed of thousands of PUs, it is impractical to consider a complex network topology that would consume superlinear area. We thus assume a linear complexity ring topology interconnection network in all three architectures, where in CSIMD the network is 32 bits wide, and in GP-SIMD and AP, the network is 1 bit wide. In a ring topology, within $O(N)$ serial steps, $N$ PUs can exchange data according to any permutation. To simplify the analysis, CSIMD, AP, and GP-SIMD analytical equations were consolidated into Table IV.

\subsection{Conventional SIMD Processor}

A CSIMD coprocessor is depicted on the left side of Figure 2. The CSIMD coprocessor contains $n_{C S I M D}$ baseline PUs, each containing a floating-point unit and a register file. The PUs are interconnected using an interconnection network. The PUs are also connected to the sequential processor shown on the right, through a bandwidthlimited interface (denoted by the red bus). Synchronization is moving data from the sequential processor to the CSIMD coprocessor before the parallel segment begins, and back from CSIMD after the parallel segment completes. Since it involves access to a shared resource, $T_{\text {Sync-CSIMD }}$ might depend on the number of PUs [Flatt et al. 1989; Reddaway 1973]. The synchronization time [Yavits et al. 2014b] can be expressed as in Equation (3), where $C_{S y n c}$ denotes the fraction of the workload that corresponds to synchronization overhead, and $B W$ denotes the bandwidth (words per second) of the interface. The execution time of the workload on the CSIMD coprocessor is thus Equation (6), where $n_{C S I M D}$ is the number of CSIMD's Pus. The speedup of the CSIMD processor over the sequential CPU can be written as in Equation (9).

The area of the CSIMD processor can be presented as in Equation (12), where $A_{A L U}$ is the ALU area and $A_{R F}$ is the register file area. The inter-PU connection network is omitted for simplicity. For efficient comparison between PU and memory areas, we represent all area values (ALU, registers, memory) in terms of baseline SRAM cell area. Let the baseline SRAM cell area be 1. In 22nm CMOS technology, the actual figure is in the range of $0.1 \mu \mathrm{m}^{2}$ [Auth et al. 2012]. Then we can write Equation (15), where $A_{C S I M D A L U}$ is the area of a single bit of a high-speed parallel ALU and $A_{C S I M D R F o}$ is the area of a register bit (a flip-flop), both measured in baseline SRAM cell area units; $m$ is data word length and $k$ is the size of the register file. This model assumes that a floating-point ALU is similar in area to a large integer multiplier. The model is quite simplistic and does not take into account numerous aspects of CSIMD design (instruction cache, communication and control, etc.). Its purpose is providing the best 
Table IV. Analytical Models

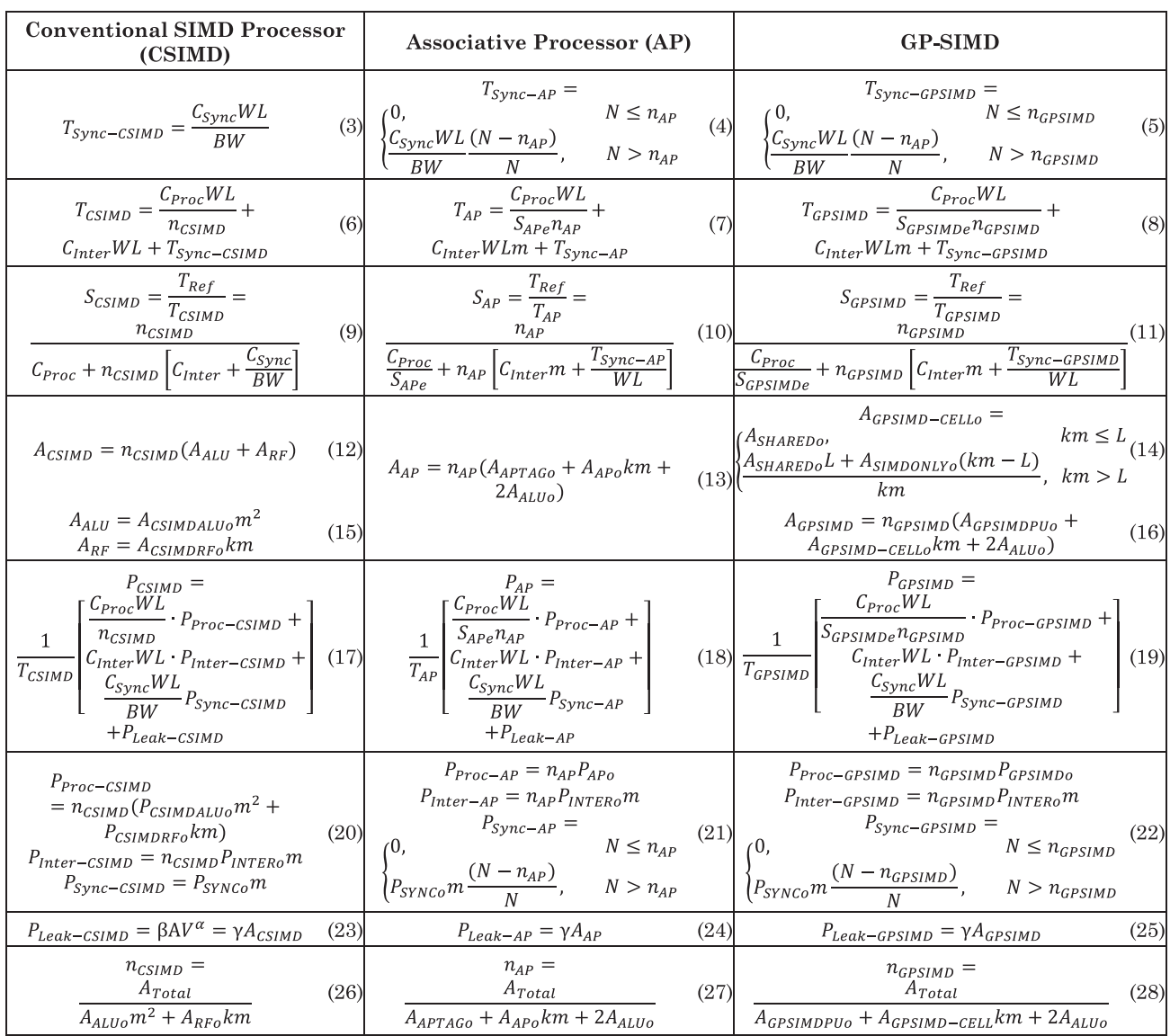

case reference figures for the comparative analysis of the CSIMD processor's speedup, area, and power.

The average power of the CSIMD processor can be written as in Equation (17), where $P_{\text {Proc-CSIMD }}$ is the average power consumed during a single processing operation, $P_{\text {Inter }}$ is the average power consumed during inter-PU communication, $P_{\text {Sync-CSIMD }}$ is the average power consumed during synchronization, and $P_{\text {Leak-CSIMD }}$ is the leakage power. Just as in the case of area comparison, we represent all power values (ALU, registers, memory) through the write power consumption of a baseline SRAM memory cell. Let the power consumption of the baseline SRAM cell during write from "0" to " 1 " or from " 1 " to "0" be 1. In $22 \mathrm{~nm}$ CMOS technology, the actual figure is in the range of $1 \mu \mathrm{W}$ at $4 \mathrm{GHz}$ [Hentrich et al. 2009]. Then we can further write the CSIMD power consumption as in Equation (20), where $P_{C S I M D A L U o}$ and $P_{C S I M D R F o}$ are the average per-bit power consumptions of the ALU and RF, respectively, during computation. $P_{I N T E R o}$ is the per-bit power consumption during the inter-PU communication. $P_{S Y N C o}$ is the per-bit power consumed during synchronization. $P_{\text {Proc-CSIMD }}, P_{\text {Inter-CSIMD }}$, and $P_{\text {Sync-CSIMD }}$ are measured in SRAM cell write power consumption units. Leakage power can be expressed as in Equation (26), where $A$ is the area, $V$ is the supply voltage, $\alpha$ and $\beta$ are constants, and $\gamma$ is the leakage area coefficient that depends on silicon process technology and operating conditions. 


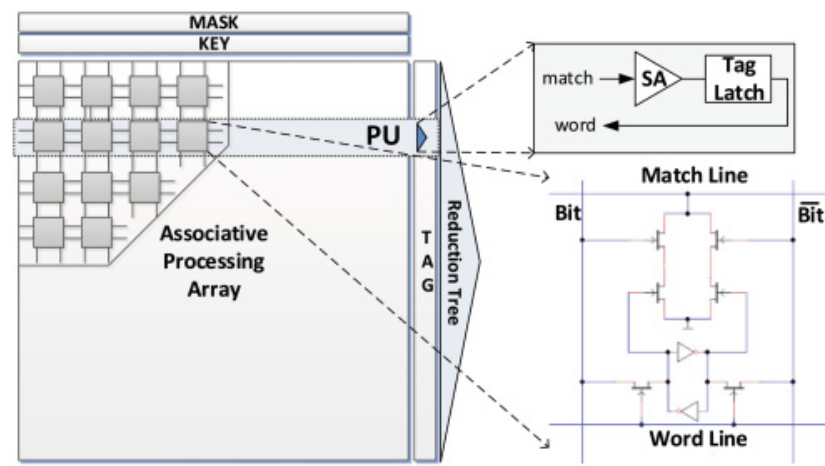

(a)

(b)

Fig. 17. AP: (a) top-level view; (b) tag logic and NOR-type bit cell.

\subsection{Associative Processor}

In this section, we detail the analytical model for the speedup, area, and power consumption of the AP. AP is based on content addressable memory (CAM), which allows comparing the stored data words to a key, tagging the matching words, and writing new data in tagged rows. AP is a non-von Neumann in-memory computing accelerator. The results of basic computing operations are precalculated and compiled into a sequence of AP instructions. The operands are stored in the CAM array. In response to an input combination, the result is written into the CAM, typically to multiple locations. The architecture of AP is presented in Figure 17(a). The associative processing array (essentially a CAM) is composed of bit cells (Figure 17(b)) organized in bit columns and word rows. Typically, a word row makes a PU. Several special registers are appended to the associative processing array. The KEY register contains a key data word to be written or compared against. The MASK register defines the active fields for write and read operations, enabling bit selectivity. The TAG register marks the rows that are matched by the compare operation and may be affected by a parallel write. An optional interconnect switch allows the PUs within the AP to communicate in parallel. A Reduction Tree is an adder tree, enabling quick parallel accumulation of TAG bits (and of an array of numbers). An example of a CMOS-based associative bit cell as well as the tag logic are shown in Figure 17(b). SA is a small sense amplifier. A detailed AP design is presented in Yavits et al. [2014a]. In a conventional CAM, a compare operation is typically followed by a read of the matched data word. In AP, a compare is usually followed by a parallel write into the unmasked bits of all tagged rows. In a similar manner to GP-SIMD, a fixed-point $m$-bit addition takes $16 m \in O(m)$ cycles. Subtraction is performed similarly and also requires $O(m)$ cycles. Fixed-point multiplication and division in AP require $O\left(\mathrm{~m}^{2}\right)$ cycles. A detailed description of AP architecture and operations can be found in Yavits et al. [2014a].

In a similar manner to GP-SIMD, the AP [Yavits et al. 2014a] does not entail data synchronization unless the entire dataset does not fit in the AP's memory array, and thus, Equation (4). The execution time of the concurrent portion of the workload can be written as in Equation (7), where $n_{A P}$ is the number of PUs in the AP, and $S_{A P e}$ is the speedup of the associative PU relative to the CSIMD's PU. Lacking a priori knowledge of the workloads to be executed on the AP, we assume the worst-case scenario consisting of a continuous series of single-precision floating-point multiplications, which in one direct implementation takes 8,800 cycles versus a single cycle on the baseline CSIMD's PU. In this case, $S_{A P E}=1 / 8800$. The speedup of the AP can then be written as in Equation (10). 
The area of the AP can be written as in Equation (13), where $k$ is the width of the associative processor memory array (in $m$-bit data words) reserved for temporary storage; $A_{A P T A G o}$ is the AP TAG cell area and $A_{A P_{0}}$ is the AP memory cell area, both measured in SRAM cell area units; and $2 A_{A L U o}$ is the per-PU hardware reduction tree size, assuming the adders of each tree level are of growing word length [Yavits et al. 2014a]. Similarly to the CSIMD coprocessor, we ignore the area of the interconnection network for simplicity.

The average power of the AP can be written as in Equation (18), where $P_{P r o c-A P}$ is the average power consumed during a single processing operation, $P_{\text {Inter-AP }}$ is the average power consumed during inter-PU communication, $P_{S y n c-A P}$ is the average power consumed during synchronization when the entire dataset does not fit in the $\mathrm{AP}$, and $P_{\text {Leak-AP }}$ is the leakage power. In a similar manner to the previous section, we can further write the AP power consumption as in Equation (21), where $P_{A P o}$ is the average per-bit power consumption of the AP during computation; $P_{I N T E R-o}$ is the perbit power consumption during the inter-PU communication; $P_{S Y N C o}$ is the per-bit power consumed during synchronization; $P_{\text {Proc-AP }}, P_{\text {Inter-AP }}$, and $P_{S y n c-A P}$ are measured in SRAM cell write power consumption units. Leakage power can be expressed as in Equation (27). Note that for comparison purposes, we use the same leakage power formulation (represented as a function of area only as in Equation (26)) for both the AP and the CSIMD processor. This may overestimate AP power somewhat: first, the leakage power per area could be lower for memory than for logic [Pollack 1999], and second, APs have fewer hotspots [Yavits et al.]. Since the leakage power is highly temperature dependent, hotspots may lead to higher leakage in the CSIMD processor [Banerjee et al. 2003]. A detailed analysis of $P_{A P_{o}}$ can be found in Yavits et al. [2014a].

\subsection{GP-SIMD Processor}

In this section, we detail the analytical model for the speedup, area, and power consumption of the GP-SIMD. As discussed in Section 3, the GP-SIMD does not entail data synchronization unless the entire dataset does not fit in the GP-SIMD's memory array, and thus, Equation (5). The execution time of the concurrent portion of the workload can be written as in Equation (8), where $n_{A P}$ is the number of PUs in the GP-SIMD, and $S_{G P-S I M D e}$ is the speedup of associative PU relative to the CSIMD's PU. Similar to the AP analysis, we assume the worst-case scenario consisting of a continuous series of single-precision floating-point multiplications, which in one direct implementation takes 2,500 cycles versus one cycle on the baseline CSIMD's PU. In this case, $S_{G P-S I M D e}=1 / 2500$. The speedup of the GP-SIMD can then be written as in Equation (11).

Following Section 3.7, we assume that the GP-SIMD's sequential processor accesses only the first $L$ bits of the memory array. Thus, the average size of the memory bit cell is in Equation (14), where $k$ is the width of the GP-SIMD memory array (in $m$-bit data words) reserved for temporary storage, and $A_{S H A R E D o}$ is the shared memory cell area and $A_{\text {SIMDONLYo }}$ is the SIMD-only memory cell area, both measured in SRAM cell area units. The total area of the GP-SIMD can be written as in Equation (16), where $A_{\text {GPSIMDPUo }}$ is the GP-SIMD processing unit cell area measured in SRAM cell area units, and $2 A_{A L U o}$ is the per-PU hardware reduction tree size. Similarly to the CSIMD coprocessor and the AP, we ignore the area of the interconnection network for simplicity.

The average power of the GP-SIMD can be written as in Equation (19), where $P_{\text {Proc-GPSIMD }}$ is the average power consumed during a single processing operation, $P_{\text {Inter-GPSIMD }}$ is the average power consumed during inter-PU communication, $P_{S y n-G P S I M D}$ is the average power consumed during synchronization when the entire dataset does not fit in the AP, and $P_{\text {Leak-GPSIMD }}$ is the leakage power. In a similar 
Table V. Model Parameters

\begin{tabular}{|c|c|c|c|}
\hline Parameter & Description & Attributed to & Value \\
\hline$\overline{A_{C S I M D A L U o}}$ & FP ALU bit cell area & CSIMD & $40^{(1)}$ \\
\hline$P_{C S I M D A L U o}$ & FP ALU bit cell power & CSIMD & $40^{(1)}$ \\
\hline$A_{A L U o}$ & ALU bit cell area & All & $10^{(1)}$ \\
\hline$P_{A L U o}$ & ALU bit cell power & All & $10^{(1)}$ \\
\hline$A_{C S I M D R F O}$ & Register bit (FF) area & CSIMD & $3^{(1)}$ \\
\hline PCSIMDRFo & Register bit (FF) power & CSIMD & $3^{(1)}$ \\
\hline$S_{A P e}$ & $\mathrm{AP}$ speedup relative to sequential CPU & $\mathrm{AP}$ & $1 / 8800$ \\
\hline$A_{A P o}$ & $\mathrm{AP}$ bit cell area & $\mathrm{AP}$ & $2^{(1)}$ \\
\hline$P_{A P o}$ & AP bit cell power & $\mathrm{AP}$ & $4^{(1)}$ \\
\hline$A_{A P T A G o}$ & AP TAG cell area & $\mathrm{AP}$ & $1^{(1)}$ \\
\hline$S_{G P S I M D e}$ & GP-SIMD speedup relative to sequential CPU & GP-SIMD & $1 / 2500$ \\
\hline$A_{S H A R E D o}$ & GP-SIMD shared bit cell area & GP-SIMD & $7 / 6^{(1)}$ \\
\hline$A_{S I M D O N L Y O}$ & GP-SIMD SIMD-only cell area & GP-SIMD & $5 / 6^{(1)}$ \\
\hline$A_{G P S I M D P U o}$ & GP-SIMD PU area & GP-SIMD & $10^{(1)}$ \\
\hline$P_{G P S I M D P U o}$ & GP-SIMD PU power & GP-SIMD & $10^{(1)}$ \\
\hline$P_{\text {INTERo }}$ & GP-SIMD intercommunication power & All & $200^{(1)}$ \\
\hline$P_{S Y N C o}$ & GP-SIMD synchronization power & All & $200^{(1)}$ \\
\hline$m$ & Data word length & All & 32 \\
\hline$k$ & Register file size (in 32-bit words) & All & 8 \\
\hline$L$ & Sequential processor word length & GP-SIMD & 64 \\
\hline$\gamma$ & Static power coefficient & All & $50 \mathrm{~mW} / \mathrm{mm}^{2}$ \\
\hline$C_{S y n c}$ & Workload portion dedicated for synchronization & All & $3 \%$ \\
\hline$C_{\text {Inter }}$ & Workload portion dedicated for intercore communication & All & $0 \%$ \\
\hline$B W$ & Bandwidth (number of $32 \mathrm{~b}$ words per cycle) & CSIMD & 4 \\
\hline
\end{tabular}

(1) Area and power parameters are relative to the area and power of SRAM bit cell respectively; the values are based on typical standard cell libraries.

manner to the previous section, we can further write the GP-SIMD power consumption as in Equation (22), where $P_{G P S I M D o}$ is the average per-bit power consumption of the AP during computation; $P_{\text {INTERo }}$ is the per-bit power consumption during the interPU communication; $P_{S Y N C o}$ is the per-bit power consumed during synchronization; and $P_{\text {Proc-GPSIMD }}, P_{\text {Inter-GPSIMD }}$, and $P_{\text {Sync-GPSIMD }}$ are measured in SRAM cell write power consumption units. Leakage power can be expressed as in Equation (28). Note that for comparison purposes, we use the same leakage power formulation (represented as a function of area only as in Equation (26)) for the CSIMD, AP, and GP-SIMD architectures. This might be somewhat unfair to the GP-SIMD: first, the leakage power per area could be lower for memory than for logic [Pollack 1999], and second, GP-SIMD, in a similar manner to AP, may have fewer hotspots [Yavits et al.]. Since the leakage power is highly temperature dependent, hotspots may lead to higher leakage in the CSIMD processor [Banerjee et al. 2003]. Note that the AP's arithmetic/logic performance [Yavits et al. 2014a] is $2.5 \times$ to $5 \times$ lower than GP-SIMD's arithmetic performance, regardless of the number of processing elements, $\mathrm{N}$.

\subsection{Performance Under Constrained Area}

Consider a given die total area, $A_{\text {Total }}$. The number of the CSIMD, AP, and GP-SIMD processing units can be found as in Equations (26) to (28). Substituting $n_{S I M D}, n_{A P}$, and $n_{G P S I M D}$ into the equations listed in the previous subsections, we determine the speedup and the power equations of each architecture. The area and power parameters we use for modeling purposes are listed in Table V.

Speedup versus area for the CSIMD coprocessor, the AP, and the GP-SIMD are shown in Figure 18(a). For example, at $2 \mathrm{~mm}^{2}$, about 500 reference floating-point engines are enabled (including registers and other logic besides the ALUs) in the CSIMD processor 
GP-SIMD, AP and CSIMD under Constrained Area
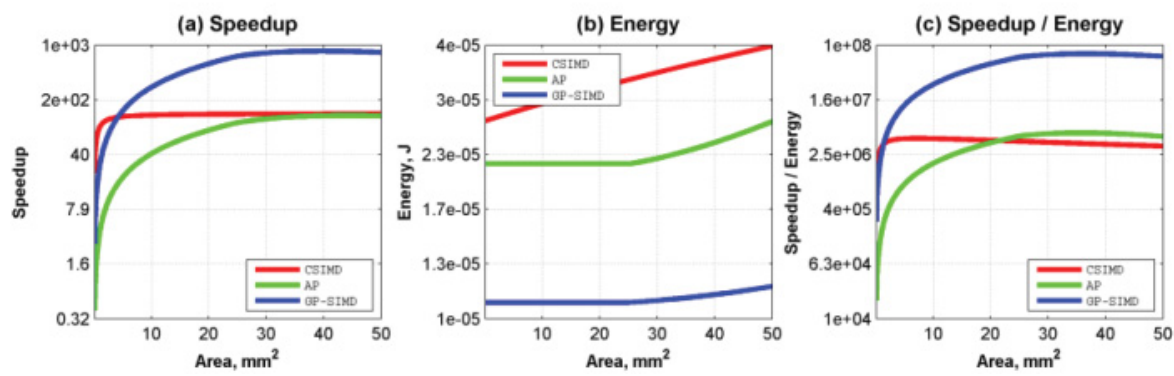

Fig. 18. Analytical results under constrained area: (a) speedup, (b) energy, (c) speedup/energy ratio.

and dissipate about $8 \mathrm{~W}$. The portion of the workload dedicated for synchronization, $C_{S y n c}$, is assumed to be 0.03 (namely, synchronization takes $3 \%$ of the workload). Note that $3 \%$ is quite conservative, as for certain workloads such as DMM (Dense Matrix Multiplication), the CPU-GPU synchronization may consume 20\% of the processing time [Volkov et al. 2008]. As the area budget increases, the speedup of the CSIMD coprocessor exhibits diminishing returns caused by synchronization, because the size of the dataset grows while the CPU $\Leftrightarrow$ SIMD bandwidth remains fixed. After sufficient area becomes available, the speedup saturates.

For smaller total area, the speedups of the AP and GP-SIMD are lower than the speedup of the CSIMD coprocessor. The breakeven point lies around $14 \mathrm{~mm}^{2}$ for the GP-SIMD. Diminishing returns affect the GP-SIMD and AP speedups to a lesser extent than in the CSIMD, since they only occur when the dataset does not fit into the internal memory array. Following Yavits et al. [2014a], to emphasize this effect, and for illustrative purpose only, we assume that the dataset size grows with the GP-SIMD and AP size, respectively, and up until $A_{\text {Total }}=25 \mathrm{~mm}^{2}$ (at which point, $n_{G P S I M D}=1.0 \times 10^{6}$ and $n_{A P}=5.3 \times 10^{5}$ ) the GP-SIMD and AP internal memory array have just enough space to hold all necessary program variables. However, from that point on, both processors will have to exchange additional data with, for example, an external DRAM. We assumed that for each additional processing unit beyond $A_{\text {Total }}=25 \mathrm{~mm}^{2}$, a single $32 \mathrm{~b}$ data word per 300 additional processing units will incur synchronization delay. These assumptions cause the GP-SIMD and AP speedup to saturate as well. Note that without this assumption, both GP-SIMD and AP speedup would have continued to climb linearly with area. Thus, off-chip bandwidth affects GP-SIMD in a similar manner to conventional GPUs. Note, however, that at $A_{T \text { otal }}=25 \mathrm{~mm}^{2}$ (just prior to saturation), the GP-SIMD provides speedup of $5.5 \times$ over the CSIMD and $7 \times$ over the AP. The speedup factor over CSIMD continues to grow till the GP-SIMD saturates at $A_{\text {Total }}=40 \mathrm{~mm}^{2}$.

Energy versus area is shown in Figure 18(b). Energy of both GP-SIMD and AP is lower than that of the CSIMD processor. Note that even when the speedups saturate, energy continues to grow with area, due to the addition of processing units and leakage. The speedup/energy ratio versus area is shown in Figure 18(c). For the CSIMD processor, the speedup/energy ratio eventually drops because the speedup saturates while energy continues to grow with increasing area. As CSIMD's speedup saturates, both GP-SIMD and AP yield better speedup/energy ratios. With further area growth, the speedup/energy ratio of both GP-SIMD and AP drops due to assumed saturation.

In general, GP-SIMD achieves higher performance than CSIMD due to CSIMD stalling on synchronization. GP-SIMD achieves higher performance per area than AP due to its lower memory cell area $(\sim 2 \times)$, allowing the integration of twice the amount 
of PUs and memory rows per a given area, and its faster floating-point implementation $(\sim 3.5 \times)$ due to GP-SIMD arithmetic PU versus associative-only PU of AP.

\subsection{GP-SIMD Cycle-Accurate Simulation}

The GP-SIMD, CSIMD, and AP high-level analytical models cannot be used for realistic simulations of known workloads but are useful for early architectural design space exploration and trend analysis. In this section, we detail a home-grown cycle-accurate simulator of GP-SIMD, useful for the design and performance evaluation of real workload. Following Yavits et al. [2014a] and Yavits et al. [2014b], four workloads have been selected for performance and energy consumption simulations:

$-N$-point fast Fourier transform (FFT)

$-N$-option pairs Black-Scholes option pricing (BSC)

$-N$-point vector reduction (VR)

-Dense matrix multiplication of two $\sqrt{N} \times \sqrt{N}$ matrices (DMM),

where $N$ is the dataset size, scaled for simplicity to the processor size (following the methodology suggested in Gunther et al. [2011] and Yavits et al. [2014c]), that is, $N=n_{G P S I M D}$. Note that simulations do not cover the cases where the data size exceeds the size of the processor (requiring data synchronization).

The workloads are hand-coded for the simulations. For FFT, we use optimized parallel implementation, outlined in Quinn [1987]. For $N$ point FFT, the sequential computing time is $O(N \log N)$. For $N$ PU parallel implementation, computing time is reduced to $\mathrm{O}(\log N)$. Following each computing step, $N$ intermediate results need to be exchanged among the PUs. We thus assign each multiply-accumulate operation to a single PU. For Black-Scholes (BSC), we used a direct implementation, based on the formulation in Black and Scholes [1973]. With a sequential computing time of $O(N)$, BSC option pricing requires no interaction between separate option calculations (BSC option pricing is an example of an "embarrassingly parallel" task). We thus assign a single PU to handle a single call option of a single security at a single strike price and a single expiration time. Vector reduction (VR) is implemented using the software reduction tree where a single PU retains a single vector element. For $\sqrt{N} \times \sqrt{N}$ dense matrix multiplication (DMM), the sequential execution time is $O\left(N^{3 / 2}\right)$. The GP-SIMD DMM algorithm utilizes $N$ PUs and yields a parallel execution time of $O(N)$, with $\sqrt{N}$ data elements being broadcast at every step [Morad et al. 2014]. The DMM algorithm employs the hardware reduction tree to accelerate multiple vector summations in parallel: at half the depth of the reduction tree, all $\sqrt{N}$ vector sums are available and are transferred directly to the sequential processor.

The simulator is cycle based, keeping records of the state of each register of each PU and of the memory row assigned to it. Each command (e.g., floating-point multiply) is broken down to a series of fine-grained single-bit PU operations. In a similar manner to SimpleScalar [Burger Austin 1997], the simulator also keeps track of the registers, buses, and memory cells that switch during execution. With the switching activity and area power models of each baseline operation detailed in Table V, the simulator tracks the total energy consumed during workload execution. We follow the area assumptions detailed in Table $\mathrm{V}$ and simulate speedup and power for growing datasets corresponding to the growing number of PUs and hence the growing total area.

Cycle-based simulated speedup is presented in Figure 19(a); speedup is defined in Equation (11). Energy consumption is shown in Figure 19(b), with DMM being the most energy-hungry benchmark. Figure 19(c) depicts the speedup/energy ratio. Note the small discontinuities in FFT and VR charts, which are due to the fact that these algorithms are designed for specific data sizes ( $N$ integral power of 2$)$. DMM shows 
GP-SIMD executing FFT, BSC, VR and DMM with cycle driven simulator
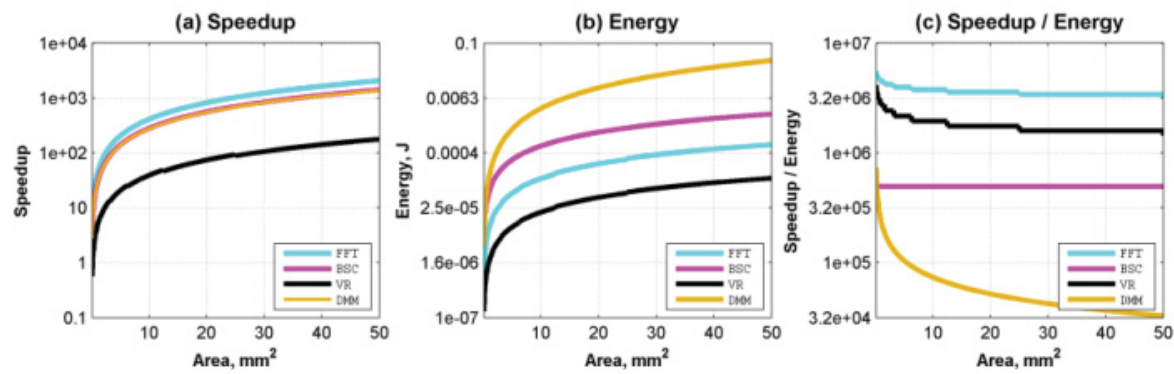

Fig. 19. Simulation results: (a) speedup, (b) energy, and (c) speedup/energy ratio for FFT, Black-Scholes, vector reduction, and dense matrix multiply algorithms.

the worst speedup/energy over all workloads. As BSC is an embarrassingly parallel workload, its speedup/energy ratio remains practically constant with growing data set size and area.

Sparse matrix multiplication (SpMM) on GP-SIMD [Morad et al. 2014] on a large set of matrices demonstrated a potentially improved power efficiency of $20 \times$ relative to a number of GPU designs. Specifically, the GP-SIMD SpMM power efficiency ranges between 0.1 and 100GFLOPS/W. The SpMM power efficiency of GPUs such as NVidia's K20 and GTX660 is in the 0.1 to $0.5 \mathrm{GFLOPS} / \mathrm{W}$ range (as reported by references cited in Morad et al. [2014]). A wide variety of multicore processors such as quad-core AMD Opteron 2214, quad-core Intel Xeon E5345, eight-core Sun UltraSparc T2+ T5140, and eight-SPE IBM QS20 Cell reportedly reach an SpMM power efficiency of up to $0.03 \mathrm{GFLOPS} / \mathrm{W}$. We should emphasize that these results were obtained through analytical modeling and cycle-accurate simulation; they have yet to be validated through real measurements on fabricated circuits.

\section{CONCLUSIONS}

GP-SIMD is a novel processor architecture that combines a general-purpose sequential processor, a massively parallel SIMD array, and a large memory shared by both processors. The shared memory may be accessed simultaneously by the sequential processor (providing a row address and accessing the data columns) and by the SIMD processor (providing a column address while each processing unit accesses a memory row). Thus, data do not need to be transferred (synchronized) back and forth between separate memories of the two processors.

GP-SIMD is compared with two other massively parallel, processing-in-memory (PIM) architectures: a conventional SIMD (CSIMD) employing separate memories for the SIMD and sequential processors and requiring data synchronization (the SIMD array composed of a moderate number of FPUs, a la GPU), and an associative processor (AP) combining storage and processing in each cell. Comparison is made by means of analytical study, and a cycle-accurate simulator is employed to validate the performance and energy of four benchmarks on GP-SIMD.

GP-SIMD speedup grows faster with area than that of CSIMD due to finer granularity of its processing unit, resulting in more processing units per equivalent area. GP-SIMD speedup also grows faster with area than that of the associative processor due to the finer granularity of its storage elements, resulting in larger memory per equivalent area. Unlike CSIMD, the performance of GP-SIMD depends on the data word length rather than dataset size. As area budget grows beyond the speedup breakeven point, the GP-SIMD's energy remains below that of the CSIMD coprocessor, and it thus 
outperforms CSIMD in terms of speedup/energy ratio over a broad spectrum of area and power budget for workloads with high data-level parallelism. In general, GP-SIMD achieves higher performance than CSIMD due to CSIMD stalling on synchronization. GP-SIMD achieves higher performance per area than AP due to its lower memory cell area, allowing the integration of twice the amount of PUs and memory rows per given area, and due to its more than twice faster floating-point implementation due to GP-SIMD arithmetic PU versus associative-only PU of AP. GP-SIMD, however, is not universally efficient. While yielding high speedup when implementing fine-grained massively data-parallel workloads (such as sparse linear algebra and machine-learning algorithms), its efficiency is much lower under workloads with low data-level parallelism, or where little or no data synchronization is required. To conclude, GP-SIMD architecture may achieve significantly higher performance and power efficiency over other conventional architectures.

\section{REFERENCES}

A. Akerib and R. Adar. 1995. Associative approach to real time color, motion and stereo vision. In Proceedings of the 1995 International Conference on Acoustics, Speech, and Signal Processing (ICASSP'95). Vol. 5. IEEE.

A. J. Akerib and S. Ruhman. 1991. Associative array and tree algorithms in stereo vision. In Proceedings of the 8th Israel Conference on Artificial Intelligence, Vision \& Pattern Recognition. Elsevier.

G. Almási et al. 2003. Dissecting Cyclops: A detailed analysis of a multithreaded architecture. ACM SIGARCH Computer Architecture News 31, 1, 26-38.

AltiVec Engine. 2014. Homepage. Retrieved from http://www.freescale.com/webapp/sps/site/overview.jsp? code=DRPPCALTVC

ARM. 2014. NEON ${ }^{\mathrm{TM}}$ General-Purpose SIMD Engine. Retrieved from http://www.arm.com/products/ processors/technologies/neon.php.

C. Auth et al. 2012. A 22nm high performance and low-power CMOS technology featuring fully-depleted tri-gate transistors, self-aligned contacts and high density MIM capacitors. In Proceedings of the 2012 Symposium on VLSI Technology (VLSIT'12). IEEE.

K. Banerjee et al. 2003. A self-consistent junction temperature estimation methodology for nanometer scale ICs with implications for performance and thermal management. Electron Devices Meeting, 2003. IEDM'03 Technical Digest. IEEE International. IEEE.

K. E. Batcher. 1974. STARAN parallel processor system hardware. In Proceedings of the National Computer Conference. 405-410.

N. Binkert et al. 2011. The gem5 simulator. ACM SIGARCH Computer Architecture News 39, 2, 1-7.

F. Black and M. Scholes. 1973. The pricing of options and corporate liabilities. Journal of Political Economy 81, 637-654.

S. Borkar. 2007. Thousand core chips: A technology perspective. In Proceedings of the ACM/IEEE 44th Design Automation Conference (DAC'07). 746-749.

J. Brockman et al. 2004. A low cost, multithreaded processing-in-memory system. In Proceedings of the 31st International Symposium on Computer Architecture.

D. T. Burger Austin. 1997. The SimpleScalar tool set, version 2.0. ACM SIGARCH Computer Architecture News 25, 3, 13-25.

A. Cassidy and A. Andreou. 2012. Beyond Amdahl Law - An objective function that links performance gains to delay and energy. IEEE Transactions on Computers 61, 8, 1110-1126.

E. L. Cloud. 1988. The geometric arithmetic parallel processor. In Proceedings of the 2nd Symposium on the Frontiers of Massively Parallel Computation. IEEE.

P. Dlugosch, D. Brown, P. Glendenning, M. Leventhal, and H. Noyes. 2014. An efficient and scalable semiconductor architecture for parallel automata processing. In IEEE Transactions on Parallel and Distributed Systems. 1-1.

J. Draper et al. 2002. The architecture of the DIVA processing-in-memory chip. In Proceedings of the 16th International Conference on Supercomputing. ACM.

H. Esmaeilzadeh et al. 2013. Power challenges may end the multicore era. Communications of the ACM 56, 2, 93-102.

H. Flatt et al. 1989. Performance of parallel processors. Parallel Computing 12, 1, 1-20. 
C. Foster. 1976. Content Addressable Parallel Processors. Van Nostrand Reinhold Company, New York.

M. Gokhale et al. 1995. Processing in memory: The Terasys massively parallel PIM array. IEEE Computer 23-31.

M. Gschwind et al. 2006. Synergistic processing in cell's multicore architecture. IEEE Micro 26, 2, $10-24$.

N. Gunther, S. Subramanyam, and S. Parvu. 2011. A methodology for optimizing multithreaded system scalability on multi-cores. Retrieved from http://arxiv.org/abs/1105.4301.

M. Hall et al. 1999. Mapping irregular applications to DIVA, a PIM-based data-intensive architecture. In Proceedings of the ACM /IEEE Conference on Supercomputing.

N. Hardavellas et al. 2011. Toward dark silicon in servers. IEEE Micro 31, 4, 6-15.

J. Hennessy and D. A. Patterson. 1996. Computer Architecture: A Quantitative Approach (2nd ed.) Morgan Kaufmann Publishers.

D. Hentrich et al. 2009. Performance evaluation of SRAM cells in 22nm predictive CMOS technology. In Proceedings of the IEEE International Conference on Electro/Information Technology.

M. Hill et al. 2008. Amdahl's law in the multicore era. IEEE Computer 41, 7, 33-38.

S. Hong and H. Kim. 2009. An analytical model for a GPU architecture with memory-level and thread-level parallelism awareness. ACM SIGARCH Computer Architecture News 37, 3.

IBM. 2005. PowerPC Vector/SIMD Multimedia Extension. Retrieved from http://math-at-las.sourceforge.net/ devel/assembly/vector_simd_pem.ppc.2005AUG23.pdf.

Intel. 2013. The Intel ${ }^{\mathbb{R}}$ Xeon Phi ${ }^{\mathrm{TM}}$ Coprocessor. Retrieved from http://www.intel.com/content/www/us/en/ high-performance-computing/high-performance-xeon-phi-coprocessor-brief.html.

S. W. Keckler et al. 2011. GPUs and the future of parallel computing. IEEE Micro 31, 5, 7-17.

P. Kogge et al. 2000. PIM architectures to support petaflops level computation in the HTMT machine. In Proceedings of the International Workshop on Innovative Architecture for Future Generation Processors and Systems.

C. E. Kozyrakis et al. 1997. Scalable processors in the billion-transistor era: IRAM. Computer 30, 9, 7578.

S. Kumar. 2012. Smart Memory. Retrieved from http://www.hotchips.org/wp-content/uploads/hc_archives/ hc22/HC22.23.325-1-Kumar-Smart-Memory.pdf.

G. Lipovski and C. Yu. 1999. The dynamic associative access memory chip and its application to SIMD processing and full-text database retrieval. In Proceedings of the IEEE International Workshop on Memory Technology, Design and Testing.

G. Loh. 2008. The cost of uncore in throughput-oriented many-core processors. In Proceedings of the Workshop on Architectures and Languages for Throughput Applications (ALTA).

D. Luebke. 2004. General-purpose computation on graphics hardware. In Proceedings of the SIGGRAPH Workshop.

T. Midwinter, M. Huch, P. A. Ivey, and G. Saucier. 1988. Architectural considerations of a wafer scale processor IEE Colloquium on VLSI for Parallel Processing 4/1, 4/4, 17.

A. Morad et al. 2013. Generalized multiAmdahl: Optimization of heterogeneous multi-accelerator SoC. Computer Architecture Letters 13, 1, 37-40.

A. Morad et al. 2014. Convex optimization of resource allocation in asymmetric and heterogeneous SoC. Power and Timing Modeling, Optimization and Simulation (PATMOS).

A. Morad et al. 2014. Efficient dense and sparse matrix multiplication on GP-SIMD. Power and Timing Modeling, Optimization and Simulation (PATMOS).

A. Morad et al. 2014. Optimization of asymmetric and heterogeneous SoC. Under review.

T. Morad et al. 2006. Performance, power efficiency and scalability of asymmetric cluster chip multiprocessors. IEEE Computer Architecture Letters 5, 1, 14-17.

J. Owens et al. 2008. GPU computing. Proceedings of the IEEE 96, 5, 879-899.

A. Pedram. 2013. Algorithm/Architecture Codesign of Low Power and High Performance Linear Algebra Compute Fabrics. PhD dissertation, University of Texas. Retrieved from http://repositories.lib. utexas.edu/bitstream/handle/2152/21364/PEDRAM-DISSERTATION-2013.pdf?sequence=1.

F. Pollack. 1999. New microarchitecture challenges in the coming generations of CMOS process technologies. Proceedings of the 32nd Annual ACM/IEEE International Symposium on Microarchitecture. IEEE Computer Society.

J. Potter et al. 1994. ASC: An associative-computing paradigm. Computer 27, 11, 19-25.

S. Pugsley et al. 2014. Comparing implementations of near-data computing with in-memory MapReduce workloads. IEEE Micro 34, 4, 44-52. 
G. Qing, X. Guo, R. Patel, E. Ipek, and E. Friedman. 2013. AP-DIMM: Associative computing with STTMRAM. In Proceedings of the Annual International Symposium on Computer Architecture (ISCA'13). ACM, New York, NY.

M. Quinn. 1987. Designing Efficient Algorithms for Parallel Computers. McGraw-Hill, 125.

S. F. Reddaway. 1973. DAP_a distributed array processor. ACM SIGARCH Computer Architecture News 2 , $4,61-65$.

B. Rogers et al. 2009. Scaling the bandwidth wall: Challenges in and avenues for CMP scaling. In Proceedings of the 36th Annual International Symposium on Computer Architecture (ISCA'09). ACM, New York, NY, 371-382.

R. M. Russell. 1978. The CRAY-1 computer system. Communications of the ACM 21, 1, 63-72.

G. E. Sayre. 1976. STARAN: An associative approach to multiprocessor architecture. Computer Architecture. Springer, Berlin.

I. Scherson et al. 1992. Bit-parallel arithmetic in a massively-parallel associative processor. IEEE Transactions on Computers 41, 10.

J. Sheaffer et al. 2005. Studying thermal management for graphics-processor architectures. ISPASS

D. Steinkraus, L. Buck, and P. Simard. 2005. Using GPUs for machine learning algorithms. IEEE ICDAR.

T. Sterling and H. Zima. 2002. Gilgamesh: A multithreaded processor-in-memory architecture for petaflops computing. In Proceedings of the ACM/IEEE Conference on Supercomputing.

J. Suh et al. 2001. A PIM-based multiprocessor system. In Proceedings of the 15th International Symposium on Parallel and Distributed Processing.

L. W. Tucker and G. G. Robertson. 1988. Architecture and applications of the connection machine. Computer $21,8,26-38$.

V. Volkov and J. W. Demmel. 2008. Benchmarking GPUs to tune dense linear algebra. In Proceedings of the 2008 ACM / IEEE Conference on Supercomputing. IEEE Press.

D. Wentzlaff et al. 2010. Core Count vs. Cache Size for Manycore Architectures in the Cloud. Technical Report. MIT-CSAIL-TR-2010-008, MIT.

L. Yavits. 1994. Architecture and Design of Associative Processor for Image Processing and Computer Vision. MSc Thesis, Technion - Israel Institute of Technology. Retrieved from http://webee.technion.ac.il/ ran/ papers/LeonidYavitsMasterThesis1994.pdf.

L. Yavits et al. 2014a. Computer architecture with associative processor replacing last level cache and SIMD accelerator. IEEE Transactions on Computers.

L. Yavits et al. 2014b. The effect of communication and synchronization on Amdahl's law in multicore systems. Parallel Computing 40.1, 1-16.

L. Yavits et al. 2014c. Thermal analysis of 3D associative processor. http://arxiv.org/abs/1307.3853v1

D. Zhang et al. 2014. TOP-PIM: throughput-oriented programmable processing in memory. In Proceedings of the 23rd International Symposium on High-Performance Parallel and Distributed Computing. ACM.

Y. Zhang and J. D. Owens. 2011. A quantitative performance analysis model for GPU architectures. In Proceedings of the 2011 IEEE 17th International Symposium on High Performance Computer Architecture (HPCA). IEEE.

Received May 2014; revised October 2014; accepted October 2014 